\title{
Dynamic chromosomal interactions and control of heterochromatin positioning by Ki67
}

\author{
Tom van Schaik ${ }^{1}$, Stefano G. Manzo ${ }^{1}$, Athanasios E. Vouzas ${ }^{2,3}$, David M. Gilbert ${ }^{2,3}$ and \\ Bas van Steensel $1^{1,4}$
}

${ }^{1}$ Division of Gene Regulation and Oncode Institute, Netherlands Cancer Institute, Plesmanlaan 121, 1066 CX Amsterdam, the Netherlands

${ }^{2}$ Department of Biological Science, The Florida State University, Tallahassee, 32304, FL, USA

${ }^{3}$ San Diego Biomedical Research Institute, 3525 John Hopkins Court, San Diego, 92121, CA, USA

${ }^{4}$ Correspondence to: b.v.steensel@nki.nl

\begin{abstract}
Ki67 forms a protective layer around mitotic chromosomes and is involved in genome positioning around nucleoli during interphase. However, a lack of genomic interaction maps has hampered a detailed understanding of the role of Ki67 in chromatin organization. We used pA-DamID to map genome-Ki67 interactions in three human cell lines and throughout the cell cycle. In mitosis, Ki67 shows a surprising preference for the distal 15-30 Mb of each chromosome. Early in interphase, when Ki67 is present in pre-nucleolar bodies, this is replaced by a pattern of large domains that overlaps with late-replicating DNA, which gradually shifts towards small chromosomes. Nucleolar perturbations indicate that these cell cycle dynamics correspond to nucleolar maturation during interphase. Furthermore, Ki67 competes with the nuclear lamina for interaction with late-replicating DNA, and controls replication timing at (peri)centromeric regions. Together, these results reveal a highly dynamic choreography of genome interactions and roles for Ki67 in genome organization.
\end{abstract}




\section{INTRODUCTION}

$\mathrm{Ki} 67$ is a chromosomal, nuclear and nucleolar protein that is widely used as a marker for cellular proliferation [reviewed in 1,2, 3]. It has been implicated in chromatin biology in various stages of the cell cycle. During mitosis, Ki67 is a key component of the perichromosomal layer (PCL) $[4,5]$, where it acts as surfactant to prevent chromosomal intermingling [6, 7]. Following anaphase, Ki67 changes from a repelling into an attracting behavior to exclude cytoplasmic proteins and compact chromosomes [8]. Early in interphase Ki67 accumulates in pre-nucleolar bodies (PNBs), which are punctate structures containing rRNA precursors and various proteins [9]. These PNBs gradually fade away as several mature nucleoli are formed [10-12]. In these mature nucleoli Ki67 is positioned specifically at the nucleolar rim.

Together with the nuclear lamina (NL), the nucleolus is a major hub for heterochromatin, as illustrated by both microscopy $[13,14]$ and genomics observations [1518]. Often, individual heterochromatic genomic loci are stochastically distributed between the $\mathrm{NL}$ and nucleoli, with variable preference for one or the other [18-20]. Additionally, disruption of one of the two structures may enhance interactions with the other [20,21], which may indicate a competitive mechanism. Interestingly, depletion of Ki67 has been shown to lead to a loss of heterochromatin around the nucleolus [22], suggesting that it may tether heterochromatin to the nucleolus.

So far, most studies of the interplay between Ki67 and chromatin have relied on microscopy observations [e.g., 5, 22, 23]. While these experiments have been highly informative, it has remained unclear how exactly Ki67 interacts with the genome throughout the cell cycle. Genome-wide interaction data would greatly enhance this understanding, and permit comparisons with other nuclear positioning data, the epigenetic landscape and functional readouts of the genome such as transcription and replication timing.

Here, we provide such data using our recently developed pA-DamID technology, which allows for visualization of protein-DNA interactions in situ and generation of genomewide interaction maps with high temporal resolution [24]. Our results uncover remarkably dynamic interactions of Ki67 with the genome of human cells, and provide insights into its roles in heterochromatin organization and replication timing. 


\section{RESULTS}

\section{pA-DamID captures genome - Ki67 interactions}

We used our recently developed pA-DamID method to profile Ki67 interactions with the genome. pA-DamID allows us to both create maps of genome-wide protein-DNA interactions with high temporal resolution, and to visualize these interactions in situ with the ${ }^{\mathrm{m} 6} \mathrm{~A}$-Tracer protein (Fig 1A) [19, 24]. Following pA-DamID with a Ki67 antibody in hTERT-RPE, HCT116 and $\mathrm{K} 562$ human cell lines, we indeed observe that ${ }^{\mathrm{m} 6} \mathrm{~A}-\mathrm{Tracer}$ binding (and hence the interaction of Ki67 with the genome) is enriched at nucleoli stained by Ki67, compared to a free Dam control (Fig 1B, C). ${ }^{\mathrm{m} 6} \mathrm{~A}-$ Tracer binding occurs mostly at the edges of nucleoli, indicating that Ki67 preferentially contacts DNA at the nucleolar periphery. However, Ki67 is not exclusively localized at nucleoli and may be locally enriched elsewhere, such as the nuclear periphery (Fig 1B, orange arrow). This overlaps with ${ }^{\mathrm{m} 6} \mathrm{~A}-$ Tracer staining, indicating that Ki67 at these sites can also engage in genome interactions. In addition, a moderate homogeneous ${ }^{\mathrm{m} 6} \mathrm{~A}-$ Tracer signal throughout the nucleus may be caused by low concentrations of DNA-interacting Ki67 in the nuclear interior, but also from non-specific antibody binding.

We then processed these ${ }^{\mathrm{m} 6} \mathrm{~A}$-tagged DNA samples for high-throughput sequencing to identify the genomic regions that interact with Ki67. We first describe results in unsynchronized cells; below we discuss the dynamics throughout the cell cycle. As described previously [24], pA-DamID utilizes a free Dam control to normalize for DNA accessibility and amplification biases [25]. After this Dam normalization we observed a striking domain-like pattern of Ki67 binding to the genome (Fig S1A). We balanced data resolution and reproducibility by using $50 \mathrm{~kb}$ averaging bins that yield acceptable Pearson correlation coefficients between independent replicate experiments in the range of 0.40 0.80 (Fig S1B, C); at smaller bin sizes the data were too noisy to be informative.

To validate these interaction maps, we used an HCT116 cell line with mClover- and AID-tagged Ki67, which allow for protein visualization and rapid protein depletion upon addition of auxin, respectively [7]. Incubation of these cells with auxin for 24 hours resulted in a near-complete depletion of mClover fluorescence, but only a partial decrease in Ki67 immunostaining signal (Fig S1D, E). This difference may be caused by the higher sensitivity of indirect immunofluorescence [7]. Genome-wide mapping of Ki67 interactions with pADamID resulted in a strong signal loss at the Ki67 interaction domains upon addition of auxin (Fig S1F, G). We assume that the remaining signals (e.g., on chr22) result from some residual Ki67 protein. These data verify that Ki67 interaction domains are specific for Ki67 and not caused by technical artifacts.

Finally, as Ki67 is a very large protein $(\sim 350 \mathrm{kDa})$, we reasoned that the location of the antibody epitope could affect the observed interaction patterns. Ki67 contains protein binding domains and a DNA binding domain, positioned at the $\mathrm{N}$ and C-terminus, respectively [reviewed in 2]. The initial antibody used was generated against a peptide sequence roughly in the middle of the protein ( 1150/3256 amino acids), so we chose two additional antibodies to target each protein end. As before, immunostaining following auxin- 
87

mediated Ki67 depletion confirms antibody specificity to Ki67 (Fig S2A). Following pADamID, only the C-terminus antibody results in ${ }^{\mathrm{m} 6} \mathrm{~A}$-Tracer enrichment around Ki67 domains (Fig S2B) and yields a genome-wide domain pattern that is similar to that of the initially used antibody (Fig S2C). With the N-terminus antibody some of this domain pattern can also be observed, but the data quality is rather poor (Fig S2D, E), possibly because the antibody epitope is too far from the DNA. These results thus show that Ki67 profiles can be reproduced with different antibodies, and are in accordance with C-terminal location of the DNA binding domain.

Combined, we conclude that application of pA-DamID results in robust genome-wide Ki67 interaction maps, although the data resolution remains limited to about $50 \mathrm{~kb}$. The ${ }^{\mathrm{m} 6} \mathrm{~A}-$ Tracer staining indicates that interactions of Ki67 with the genome are enriched near nucleoli, but may also occur elsewhere.

\section{Ki67 binding varies between cell types, but is consistently enriched at small chromosomes and near centromeres}

Differences in Ki67 interactions among cell types are most apparent on large chromosomes (Fig 1D, S3A). In contrast, interactions are more consistent on small chromosomes (Fig S3A), and more frequent as illustrated by a higher dynamic range compared to larger chromosomes (Fig 1E). Small chromosomes are typically positioned in the nuclear interior and in the vicinity of nucleoli $[26,27]$, suggesting that these interactions involve nucleolar Ki67.

Nucleoli are formed around rDNA repeats that are positioned on the p-arm of several human chromosomes, next to the centromere. We therefore expected to find Ki67 binding near these regions. Indeed, rDNA-containing chromosomes often have the highest Ki67 interaction scores, even though sections of these chromosomes are depleted for Ki67 (indicated by negative values in the boxplots) (Fig 1E). However, Ki67 may lack affinity for rDNA sequences themselves as these show no enrichment in our data (Fig S3B). Ki67 interactions are enriched up to several Mb outside centromeres for all cell types (Fig 1F). While this scales with chromosome size and partially with rDNA presence, sequences near centromeres are enriched for Ki67 interactions on all chromosomes (Fig 1G).

\section{Cell cycle dynamics of Ki67 interactions}

The interpretation of the Ki67 interaction profiles could be confounded by the variable location of Ki67 during the cell cycle. Microscopy studies have shown that Ki67 coats chromosomes during mitosis, localizes to pre-nucleolar bodies (PNBs) in early G1 and slowly transfers to nucleoli during interphase [4, reviewed in 28]. We hypothesized that these transitions would be accompanied by a shift in genomic interactions. To test this, we synchronized RPE cells in metaphase and harvested cells at several time points during the cell cycle to profile Ki67 interactions [24].

We first prepared cells for ${ }^{\mathrm{m} 6} \mathrm{~A}-\mathrm{Tracer}$ microscopy to validate the localization of $\mathrm{Ki} 67$ and its DNA interactions. Cells synchronized in metaphase show clearly condensed chromosomes with Ki67 and ${ }^{\mathrm{m} 6} \mathrm{~A}$-Tracer signals at the periphery of the chromosomes (Fig 
117 2A), although individual chromosomes are difficult to distinguish after pA-DamID,

118 presumably due to several hours of nuclear permeabilization without fixation (c.f. Fig $2 \mathrm{~A}$ and

119 3A). One hour after synchronization, when cells have entered interphase, Ki67 is spread

120 throughout the nucleus in numerous PNBs (Fig 2A). Later time points show a gradual

121 repositioning of Ki67 from small PNBs to several mature nucleoli. At all interphase time

122 points ${ }^{\mathrm{m} 6} \mathrm{~A}-$-Tracer signal is enriched near Ki67 (Fig 2A, B), which indicates that a

123 redistribution of Ki67 also affects its DNA interactions.

124 We then processed these samples for genome-wide mapping of the ${ }^{\mathrm{m} 6} \mathrm{~A}$-tagged Ki67

125 interaction sites. For subsequent quantitative analyses of Ki67 interactions, we first

126 converted the $\log _{2}$-ratios to z-scores. This equalizes any variable dynamic ranges between

127 conditions and replicates without affecting the data distribution, similar to previous work for

128 Lamin B1 interactions during the cell cycle [24]. The genome-wide mapping data reveal

129 several interesting patterns (Fig 2C). First, during mitosis ( $\mathrm{t}=0 \mathrm{~h}$ ) Ki67 interactions are

130 enriched at the distal ends of the chromosome ends (Fig 2C, D). The enrichment extends up

131 to $15-30 \mathrm{Mb}$ from the telomere and scales with chromosome size. Second, early in G1 phase

$132(\mathrm{t}=1 \mathrm{~h}), \mathrm{Ki} 67$ interactions are more evenly distributed among all chromosomes compared to

133 unsynchronized cells, with increased and decreased interactions for large and small

134 chromosomes, respectively (Fig 2E). Third, during interphase, Ki67 interactions are

135 gradually lost on large chromosomes in favor of smaller chromosomes (Fig 2C, F).

136 Remarkably, chromosome $X$ does not follow these trends. This may be caused by active

137 repositioning of the inactive chromosome $X$ to nucleoli during S-phase $[29,30]$. Because

138 mitosis is relatively quick and nucleoli mature in a few hours, Ki67 profiles from

139 unsynchronized cells are probably strongly enriched for interactions with mature nucleoli.

140 These changes in genome-wide binding patterns roughly coincide with the sequential

141 repositioning of Ki67 from the chromosomal periphery to PNBs, and then to mature nucleoli.

142 To further investigate the link between Ki67 dynamics during interphase and the nucleolar

143 maturation from PNBs, we disrupted nucleoli in hTERT-RPE cells with a short osmotic

144 shock. Upon recovery, the process of PNB formation and nucleolar maturation is repeated

145 [31]. Indeed, we find that Ki67-containing PNBs are strongly enriched after 30 minutes of

146 recovery and mostly lost again after 180 minutes (Fig S4A). pA-DamID maps from these

147 cells show that the 30-minute time point mostly resembles early $\mathrm{G} 1$, while the 180-minute

148 time point is more similar to late G1. Similar to early interphase, this transition corresponds

149 to an increase of Ki67 interactions on small chromosomes (Fig S4B, C). Thus, we conclude

150 that cell cycle dynamics of Ki67 interactions correspond to nucleolar maturation during

151 interphase.

152

153 Release of Ki67 from nucleoli drastically changes its DNA interactions

154 The results presented so far suggest that Ki67 has a binding potential for all chromosomes,

155 but its distribution is dynamically influenced by its cell cycle regulated interactions with PNBs

156 and nucleoli. To test the importance of nucleolar confinement further, we released Ki67 from

157 nucleoli by adding a low dose of actinomycin $D$ (ActD; $50 \mathrm{ng} / \mathrm{mL}$ ) for 3 hours. ActD at this

158 concentration specifically inhibits Poll transcription and results in nucleolar breakdown [20, 
32]. As a result, Ki67 is no longer restricted to nucleoli and shows distinct patterns from other nucleolar markers (i.e. MKI67IP) (Fig 3A, S4D-E). Ki67 localization to mitotic chromosomes is not affected by ActD (Fig 3A, orange arrow).

pA-DamID mapping shows that this nucleolar breakdown affects Ki67 binding to the genome, but in different ways depending on the cell type (Fig 3B). In hTERT-RPE and HCT116 cells, the genomic preference of Ki67 seems mostly maintained but interactions show strong quantitative differences (Fig 3B). This includes an overall shift of Ki67 from small chromosomes to large chromosomes (Fig 3C). In contrast, the interaction pattern in K562 cells is more broadly altered (Fig 3B), although a reduction of Ki67 interactions with rDNA-containing chromosomes is shared with the other two cell types (Fig 3C). All three cell types also exhibit a consistent loss of Ki67 interactions near centromeres, again most clearly for rDNA-containing chromosomes (Fig 3D). Combined, these results illustrate that nucleolar integrity is required for a normal Ki67 interaction pattern across the genome, in particular for the preference at small and rDNA-containing chromosomes.

\section{Ki67 and Lamin B1 interactions partially overlap and together mark the late-replicating genome}

There is at least a partial redundancy of heterochromatin positioning between nucleoli and the NL [reviewed in 33, 34]. To determine whether we could also observe this in our data, we compared our pA-DamID maps of Ki67 and Lamin B1 [24]. Overall, both proteins interact with mostly similar genomic domains, although their relative interaction frequencies vary strongly (Fig 4A). Often, among the domains bound by both proteins, Ki67 and Lamin B1 seem anti-correlated, where domains with the highest Ki67 signals are generally lower in Lamin B1 signals, and vice-versa (Fig 4A, orange arrows). This partial anti-correlation is visible as the "head" of a hammer-shaped scatter plot, particularly in hTERT-RPE cells (Fig 4B, top panels).

Late replicating DNA is known to be enriched at both the nuclear periphery and nucleolus [reviewed in 35]. Indeed, analysis of published maps of replication timing [36] showed strong overlap of late-replicating domains with both the Ki67 and Lamin B1 domains (Fig 4A). Strikingly, the late-replicating regions overlap almost perfectly with the hammerheads in the Ki67 versus Lamin B1 scatter plots (Fig 4B, middle panels). Thus, virtually all late-replicating domains are covered by either of the two proteins, and among these domains competition may lead to a variable balance between the two proteins.

\section{Ki67 competes with the nuclear lamina for heterochromatin in HCT116 cells}

To test this competition, we used the HCT116 Ki67-AID cell line and mapped Lamin B1 interactions following 24 hours of Ki67 depletion. Additionally, given the roles of Ki67 in mitosis and the reorganization of the genome afterwards, we combined Ki67 depletion with two cell synchronization strategies. First, Ki67 was depleted during a double thymidine block. These cells undergo a single cell division and the synchronization prevents any cell cycle perturbation that Ki67 depletion may induce. Second, to specifically test whether mitosis is required for DNA repositioning, Ki67 was depleted in G2-arrested cells using a CDK1 
inhibitor (Fig 5A). Flow cytometry confirmed cell synchronization with and without Ki67 depletion (Fig 5A, bottom panels). We first verified the Ki67 pA-DamID patterns in synchronized cells with normal Ki67 levels (i.e., not treated with auxin). Here, we found a shift of Ki67 between S-phase and G2 cells that correlates with chromosome size (Fig 5B), akin to the progressive shift towards smaller chromosomes that we observed during interphase in hTERT-RPE cells (Fig 2C, F).

Ki67 depletion induces small quantitative differences in Lamin B1 interactions (Fig $5 \mathrm{C}$, orange arrows). Importantly, increases in Lamin B1 interactions are most prominent in regions that were originally bound by Ki67 (Fig 5D). This effect is most apparent in S-phase cells (which had progressed through mitosis), but is still present in cells blocked in G2 (with Pearson correlations between the initial Ki67 score and Lamin B1 difference of approximately 0.3 and 0.2 for the two conditions, respectively). Passage through mitosis is thus not required to change genome positioning. We conclude that Ki67 counteracts Lamin B1 interactions of a subset of late-replicating domains.

\section{Ki67 promotes timely replication of centromeres}

Next, we asked whether Ki67 depletion affected the replication timing of the regions that it interacts with. We tested this by Repli-seq [37], 24 hours after Ki67 depletion in (unsynchronized) HCT116 cells (Fig 5E, F). While replication timing across most of the genome remained unaffected by this depletion, we did observe a small but consistent delay in replication timing in peri-centromeric regions (Fig 5G, H). Centromeric sequences, which cannot be uniquely mapped to individual chromosomes, also showed a delayed replication timing upon Ki67 depletion (Fig S5A). The replication timing observations are different from Lamin B1 interactions that increased near centromeres but also on other domains with high Ki67 scores (Fig 5E, purple arrows, S5B, C). Thus, Ki67 specifically regulates the replication timing of (peri-)centromeric regions.

\section{No evidence for direct gene repression by Ki67}

Genes in late replicating and NL-associated domains generally show little transcriptional activity [15, reviewed in 35, 38-40]. Indeed, mRNA-seq data show that genes in the abovementioned scatterplot hammer-head predominantly are lowly transcribed (Fig S5D). In line with this, genes with high levels of Ki67 tend to be inactive, particularly in hTERT-RPE and HTC116 cells.

We then asked whether Ki67 contributes directly to the transcriptional repression of these genes. Several studies have found changes in gene activity upon Ki67 loss [22, 30, $41,42]$, but none could attribute this to direct effects, in part because the binding pattern of Ki67 was not known. We therefore generated RNA-seq data in the HCT116 Ki67-AID cells. We synchronized these cells in S-phase, to rule out any cell cycle effects that Ki67 depletion may have in these cells (Fig 5A). Intriguingly, we find that gene expression is largely unaffected by acute Ki67 depletion, with only three genes reaching our significance cut-off: ALDH1A3, CYP1A1 and HAS3 (Fig S5E). We cannot link these genes to initial Ki67 binding 
or Ki67 biology. A similar result was obtained in a recent study that also employed auxinmediated Ki67-AID depletion in HCT116 cells [42].

We also compared Ki67 interactions with gene expression data following Ki67 depletion in hTERT-RPE cells [30]. Unlike HTC116, these cells express p21, which causes cell cycle perturbations in response to Ki67 loss, and concomitant differential expression of thousands of genes [30]. Our re-analysis of these data indicates that the changes in gene expression do not correlate with initial Ki67 binding (Fig S5F). This further indicates that $\mathrm{Ki} 67$ binding is not directly involved in gene silencing.

\section{Ki67 binding correlates positively with H3K9me3 and negatively with H3K27me3}

Finally, we compared the Ki67 patterns to those of the main repressive histone modifications H3K27me3 and H3K9me3 (Fig 6A). Remarkably, these marks are strongly correlated with the balance between Ki67 and Lamin B1. In hTERT-RPE and HCT116 cells the scatterplot hammer-head shows a strong partitioning, with Ki67 interactions being positively linked to H3K9me3 but not H3K27me3 (Fig 6B). In K562 cells the preference of H3K9me3 for Ki67rich regions is less pronounced.

\section{Following acute Ki67 depletion in HCT116 cells (Fig 5), H3K27me3 and H3K9me3} are only subtly affected (Fig 6C). The effect sizes seem smaller than for Lamin B1 interactions. Interestingly, we observed a mild increase in H3K27me3 levels for regions originally bound by Ki67 (Fig 6D), suggesting that Ki67 interactions suppress H3K27me3 deposition. The effect of Ki67 depletion on H3K9me3 is more complicated. Initial Ki67 binding is weakly correlated with an increase in $\mathrm{H} 3 \mathrm{~K} 9 \mathrm{me} 3$ in G2-synchronized cells, but we do not observe this in cells in S-phase (Fig 6D).

\section{DISCUSSION}

Microscopy studies have illustrated that Ki67 and nucleoli are important contributors to genome organization, in particular for heterochromatin positioning and the separation of mitotic chromosomes [5-8, 22]. However, a lack of genome-wide interaction profiles of Ki67 has hampered a detailed understanding of how this protein shapes and regulates the genome. In this work, we applied pA-DamID to profile genome - Ki67 interactions in several human cell lines and throughout the cell cycle.

One of the fascinating aspects of Ki67 is its role on mitotic chromosomes, where it establishes the peri-chromosomal layer $[4,5,43,44]$ and acts as a surfactant that prevents chromosomal intermingling [6, 7]. Surprisingly, our pA-DamID data indicate that Ki67 does not coat mitotic chromosomes homogeneously, but instead shows a preference for the distal $\sim 15-30 \mathrm{Mb}$ of each chromosome arm. We cannot rule out that Ki67 is selectively lost from the proximal regions during the permeabilization step that is part of the pA-DamID protocol. For example, Ki67 shows increased mobility during mitosis [45], and prolonged permeabilization may select for a more stable binding population. Because the interaction frequencies reported by $\mathrm{pA}-\mathrm{DamID}$ are relative rather than absolute, we emphasize that 
these results do not imply that the central chromosomal regions are completely devoid of Ki67, only that their occupancy is lower.

The enrichment of Ki67 at the distal regions may be caused by encapsulation of the ends of condensed chromosomes, which would result in a local increase of surface area and thus a relative increase in Ki67 signal. An alternative and not mutually exclusive explanation is that the chromosomal ends are typically facing away from the chromosomal territory [46], and thereby recruit more Ki67. This hypothesis is supported by our previous observations that the distal ends of chromosomes (up to $\sim 25 \mathrm{Mb}$ ) are enriched for Lamin B1 interactions in early $\mathrm{G} 1$ [24], suggesting that these regions are at the periphery of the chromosomal territory upon establishment of the NL. Similarly, LAP2 $\alpha$ has been reported to associate with the ends of mitotic chromosomes [47]. Possibly, Ki67 is enriched at mitotic chromosome ends because its surfactant activity is particularly critical to prevent telomere-telomere fusions. In support of this hypothesis, chromosome bridges - which are typical of such fusions - have been detected in mitotic cells that lack Ki67, although DNA damage elsewhere may also cause such bridges [42].

Following mitosis, we observed a rapid rearrangement of Ki67-DNA interactions across the genome. Within $\sim 1$ hour, Ki67 moves to many late-replicating domains. This transition may be triggered by dephosphorylation of Ki67 [45, 48]. Our data are in accordance with early microscopy observations that Ki67 overlaps with most large heterochromatin domains in early interphase [49]. However, our results also show that Ki67 gradually redistributes during early $\mathrm{G} 1$ from large to smaller chromosomes. This redistribution roughly coincides with the relocation of Ki67 from PNBs to nucleoli, and is reverted by perturbations that release Ki67 from nucleoli and re-establish PNBs. We therefore propose that Ki67 in PNBs interacts with late-replicating domains throughout the genome, while upon sequestration of Ki67 at nucleoli these interactions become restricted to small chromosomes. In support of this model, small chromosomes tend to be positioned near nucleoli $[27,50]$.

Our results implicate $\mathrm{Ki} 67$ as one of the proteins that modulate the distribution of latereplicating DNA between nucleoli and the NL [reviewed in 33, 34, 40]. Most likely this is due to direct interaction of Ki67 with a subset of late-replicating regions. It is interesting to note that both Ki67 and lamins preferentially bind AT-rich DNA in vitro [51, 52], which matches observations that both nucleolus- and lamina-associated domains are AT-rich [16, 53]. However, it is likely that other proteins besides Ki67 mediate nucleolar interactions. For example, different from our Ki67 profiles, a subset of nucleolar interactions shows enrichment for H3K27me3 and early-replicating DNA [18]. Moreover, Ki67 is slowly degraded in $\mathrm{G} 1$ and completely absent in senescent cells $[43,54,55]$, yet these cells largely maintain their nucleolus - genome interactions [17]. Exceptions are alpha-satellite sequences (at centromeres) and H3K9me3-marked chromatin, which lose nucleolar positioning upon senescence [17]. Our data show that these regions strongly interact with Ki67, supporting a direct role in nucleolus tethering.

Upon Ki67 depletion, we observed a surprising delay in replication timing specifically at (peri-)centromeres. These regions show increased Lamin B1 interactions, yet other 
regions that reposition towards the $\mathrm{NL}$ are not affected in their replication. Rather, we speculate that Ki67 directly affects replication of centromeres, which are known for their early and tightly regulated DNA replication [reviewed in 56, 57]. The perturbed replication may be consequence of increased transcription of alpha-satellite sequences following Ki67 depletion [58]. Resulting transcription-replication conflicts could result in delayed and incomplete DNA replication, as was recently shown following rapid CENP-A depletion [59].

\section{METHODS}

\section{Experimental methods}

\section{Cell culture}

hTERT-RPE cells (ATCC CRL-4000), HCT116 cells (ATCC CCL-247) and K562 cells (ATCC CCL-243) were cultured according to their ATCC handling procedure, except that cells were passaged using $0.05 \%$ Trypsin-EDTA (Gibco) instead of $0.25 \%$. Adherent cells (hTERT-RPE cells and HCT116 cells) were harvested with $0.05 \%$ Trypsin-EDTA, which was inactivated with complete medium.

HCT116 Ki67-miniAID-mClover cells (Ki67-AID in short) were kindly provided by M. Takagi and cultured as wildtype HCT116 cells. Ki67 depletion was induced by adding 500 $\mu \mathrm{M}$ auxin (from a $1 \mathrm{mM}$ stock solution) (Sigma-Aldrich), or a similar amount of DMSO for control cells.

All cells were tested for mycoplasma presence every 2 months.

\section{Cell cycle synchronization}

Cell cycle synchronization of hTERT-RPE was performed as described previously [24]. Cells were cultured for $24 \mathrm{~h}$ in $75 \mathrm{~cm}^{2}$ flasks to reach $\sim 50 \%$ confluency, after which mitotic cells were enriched for using a sequential synchronization strategy of $\mathrm{G} 2$ arrest (16 h of $10 \mu \mathrm{M}$ RO-3306, CDK1 inhibitor) followed by metaphase blocking ( $1.5 \mathrm{~h}$ of $25 \mathrm{ng} / \mathrm{mL}$ nocodazole, microtubule inhibitor). Mitotic cells were harvested by shake-off and immediately processed for pA-DamID or replated in complete medium for interphase time points. Light microscopy confirmed successful mitotic synchronization, and adherence and cell division of the replated cells. Replated cells were harvested after 1, 3, 6 and $10 \mathrm{~h}$.

\section{Osmotic shock}

hTERT-RPE cells were cultured overnight in multiple $10 \mathrm{~cm}$ dishes with $10 \mathrm{~mm}$ cover slips. Cells were washed in pre-warmed 1x phosphate-buffer saline (PBS), after which nucleoli were disrupted by a 15-minute osmotic shock (0.2x PBS) [31]. Cells were released in complete medium and harvested for pA-DamID after 30 and 180 minutes. To verify nucleolar reassembly, cover slips with attached cells were removed before harvesting, washed in PBS and fixed for 10 minutes in $2 \%$ formaldehyde/PBS. 


\section{Actinomycin D treatment}

hTERT-RPE cells, HCT116 cells and K562 cells were cultured overnight in two $10 \mathrm{~cm}$ dishes with $10 \mathrm{~mm}$ cover slips. Poll transcription was specifically inhibited using a $3 \mathrm{~h}$ treatment of $50 \mathrm{ng} / \mathrm{mL}$ actinomycin $\mathrm{D}$ (from a $1 \mathrm{mg} / \mathrm{mL}$ stock solution). A similar amount of DMSO was added to control cells. Cover slips were removed and cells were fixed with $2 \%$ formaldehyde/PBS to verify nucleolar disruption and Ki67 redistribution. For K562 cells, the cover slips were coated with poly-L-lysine to immobilize the cells. Remaining cells were harvested for pA-DamID experiments.

\section{HCT116 Ki67-AID synchronization experiments}

HCT116 Ki67-AID (M. Takagi) were synchronized using a sequential arrest into S-phase and G2-phase. First, cells were cultured overnight in $2.5 \mathrm{mM}$ thymidine to block cells in S-phase, and then washed two times in pre-warmed PBS and once in complete medium. For S-phase cells, the cells were released in complete medium for $8 \mathrm{~h}$, after which they were incubated with $2.5 \mathrm{mM}$ thymidine for another $16 \mathrm{~h}$. For G2-phase cells, the cells were released on complete medium supplemented with $10 \mu \mathrm{M}$ RO-3306 (CDK1 inhibitor) for $24 \mathrm{~h}$. During the last $24 \mathrm{~h}, \mathrm{Ki} 67$ depletion was induced by adding $500 \mu \mathrm{M}$ auxin (Sigma-Aldrich), or a similar amount of DMSO for control cells. For all conditions, cells were cultured in $10 \mathrm{~cm}$ dishes with $10 \mathrm{~mm}$ poly-L-lysine coated coverslips. Before harvesting cells for PA-DamID, cover slips were removed, washed in PBS and cells were fixed for 10 minutes with $2 \%$ formaldehyde/PBS to verify Ki67 depletion. Additionally, cells synchronization was confirmed using ethanol fixation and DNA staining with $1 \mu \mathrm{g} / \mathrm{mL}$ DAPI [37]. DNA content was measured using flow cytometry on an Attune NxT (Invitrogen, Thermo Fisher Scientific).

\section{pA-DamID}

pA-DamID experiments were performed as described previously [24]. One million cells were collected by centrifugation in $1.5 \mathrm{~mL}$ Eppendorf tubes ( 3 minutes, $500 \mathrm{~g}$, at $4^{\circ} \mathrm{C}$ ), washed in $400 \mu \mathrm{L}$ of ice-cold PBS and subsequently washed in $400 \mu \mathrm{L}$ of ice-cold digitonin wash buffer (DigWash) (20 mM HEPES-KOH pH 7.5, $150 \mathrm{mM} \mathrm{NaCl,} 0.5 \mathrm{mM}$ spermidine, 0.02\% digitonin, cOmplete Protease Inhibitor Cocktail (Roche \# 11873580001)). Cells were resuspended in $200 \mu \mathrm{L}$ DigWash supplemented with a primary antibody and rotated for 2 hours at $4{ }^{\circ} \mathrm{C}$. After a DigWash wash step, cells were resuspended in $200 \mu \mathrm{L}$ DigWash supplemented with 1:200 pA-Dam protein ( 40 units of Dam activity) and rotated for 1 hour at $4{ }^{\circ} \mathrm{C}$. After two wash steps with DigWash, Dam activity was induced by resuspension in $100 \mu \mathrm{L}$ DigWash supplemented with $80 \mu \mathrm{M}$ S-adenosyl-methionine (SAM) (NEB, B9003S) and incubated at $37^{\circ} \mathrm{C}$ for 30 minutes. For every experimental condition, one million cells were also processed without antibodies or pA-Dam protein, but instead incubated with 4 units of Dam enzyme (NEB, M0222L) during the activating step. This freely diffusing Dam moiety serves as control to account for DNA accessibility and amplification biases.

For the primary antibody incubation, we used 1:100 dilutions for antibodies against Ki67 (Abcam ab15580, rabbit), N-terminal Ki67 (Human Protein Atlas HPA001164-25UL, rabbit), C-terminal Ki67 (Novus NB600-1252-0.1 ml, rabbit), H3K27me3 (CST C36B11, 
rabbit), and H3K9me3 (Diagode C15310193, rabbit). A 1:400 dilution was used for the antibody against Lamin B1 (Abcam ab16048, rabbit).

At this point, either genomic DNA was isolated and processed for high-throughput sequencing, or cells were diluted in PBS and plated on poly-L-lysine coated cover slips for 30 minutes, followed by 10 minutes fixation with $2 \%$ formaldehyde/PBS and immunostaining. These cover slips were first coated with $0.1 \%$ (w/v) poly-L-lysine (Sigma-Aldrich, \#P8920) for 15 minutes, washed with $\mathrm{H}_{2} \mathrm{O}$ (once) and PBS (3 times) and stored in $70 \%$ ethanol for later use. Samples were prepared for single-end 65 bps Illumina Hi-seq sequencing as described previously [60, 61], except that the Dpnll digestion was omitted. Approximately 15 million reads were sequenced for every Ki67 and Dam control sample to ensure high quality Ki67 interaction data, and approximately 5 million reads were sequenced for Lamin B1, H3K27me3 and H3K9me3 libraries.

\section{Repli-seq}

Following $24 \mathrm{~h}$ of Ki67 depletion in HCT116 Ki67-AID cells (see above), BrdU was added to a final concentration of $100 \mu \mathrm{M}$ and cells were incubated at $37^{\circ} \mathrm{C}$ for another $2 \mathrm{~h}$. Cells were fixed in ice-cold ethanol and processed for Repli-seq as described [37]. Libraries were sequenced with approximately 10 million 150 bps paired-end reads per sample on an Illumina NovaSeq 6000.

\section{RNA-seq}

HCT116 Ki67-AID cells were synchronized in S-phase with $24 \mathrm{~h}$ of Ki67 depletion (see above). RNA was isolated using the Qiagen RNeasy column purification kit, after which sequencing libraries were prepared using the Illumina TruSeq polyA stranded RNA kit. Libraries were sequenced with approximately 25 million 2x54 bps paired-end reads on an Illumina NovaSeq 6000.

\section{Immunostaining and microscopy}

Cover slips with formaldehyde fixed cells were washed with PBS, permeabilized with $0.5 \%$ NP40/PBS for 20 minutes and blocked with 1\% BSA/PBS for $1 \mathrm{~h}$. Cover slips were incubated with primary antibodies at room temperature for $1 \mathrm{~h}$, and washed three times with PBS. Next, cover slips were incubated with secondary antibodies at room temperature for 45 minutes. After one wash with PBS, DNA was stained with $1 \mu \mathrm{g} / \mathrm{mL}$ DAPI for 10 minutes, followed by two washes with $\mathrm{PBS}$ and one with $\mathrm{H}_{2} \mathrm{O}$. Cover slips were mounted with Vectashield and sealed with nail polish.

Cells processed with Ki67 pA-DamID were first incubated with an antibody against Ki67 (1:1000, Abcam ab15580, rabbit), and then with ${ }^{\mathrm{m} 6} \mathrm{~A}$-Tracer protein (1:500, 1.15 $\mathrm{mg} / \mathrm{mL}$ ) and an Alexa594-fused antibody against rabbit (1:250, Jackson 711-585-152, donkey). Cells that received the osmotic shock or actinomycin $D$ were first incubated with antibodies against MKI67IP (1:500, Human Protein Atlas AMAB90961, mouse) and Ki67 (1:1000, Abcam ab15580, rabbit), and then with an Alexa488-fused antibody against mouse (1:1000, ThermoFisher A11001, mouse) and an Alexa594-fused antibody against rabbit 
(1:250, Jackson 711-585-152, donkey). HCT116 Ki67-miniAID-mClover cells were first incubated with an antibody against Ki67 (1:1000, Abcam ab15580, rabbit), and then an Alexa594-fused antibody against rabbit (1:250, Jackson 711-585-152, donkey).

Single 1,024 $\times 1,024$ pixel confocal sections around the middle plane of nuclei were imaged on a Leica SP5 with a $63 \times$ NA 1.47 oil immersion objective, using bidirectional scanning, and $8 \times$ line averaging. An additional $3 \times$ electronic zoom was used for images that required a higher magnification. Alternatively, z-stacks were imaged using $2 x$ line averaging and a $380 \mathrm{~nm}$ step size.

460

\section{Computational analyses}

462

\section{pA-DamID processing}

464

pA-DamID reads were filtered to contain the Dpnl adapter sequence, which was then trimmed off with cutadapt 1.11 and custom scripts. The remaining genomic DNA sequence was mapped to a hybrid genome consisting of GRCh38 v15 (without alternative haplotypes) and a ribosomal model (GenBank: U13369.1) with bwa mem 0.7.17. Further data processing was performed with custom $\mathrm{R}$ scripts. Reads were filtered to have a mapping quality of at least 10 and overlap with the ends of a GATC fragment, and subsequently counted in genomic bins of different sizes. In this manuscript, $50 \mathrm{~kb}$ genomic bins were used as a compromise between resolution and reproducibility of Ki67 interaction profiles.

Counts were normalized to 1 million reads and a $\log _{2}$-ratio was calculated over the Dam control sample. At least two biological replicates were generated for each condition, which ratios were averaged for downstream analyses. For quantitative comparisons, the $\log _{2}$-ratios were converted to z-scores to account for differences in dynamic range between conditions and replicates [24].

477

\section{Repli-seq}

479 Adapters and low-quality reads were removed from Repli-seq reads with fastp 0.12.2 [62].

480 Trimmed reads were mapped and processed as $\mathrm{pA}$-DamID reads with several differences.

481 First, duplicated reads were removed. Second, reads were not required to overlap with the 482 ends of GATC fragments. Third, $\log _{2}$-ratios of early/late were quantile normalized instead of 483 z-score normalization.

\section{RNA-seq}

486 Adapter sequences and low-quality reads were removed from RNA-seq reads with fastp 0.12 .2 [62]. Trimmed reads were aligned to GRCh38 v15 and counted in gencode v24 genes with STAR 2.5.4a [63]. DESeq2 1.30.1 was used to call differentially expressed genes [64], which we defined as genes that have a Benjamini-Hochberg corrected p-value lower than 0.05 when testing for $a \log _{2}$-fold change different than 0 . FPKM values were calculated with 'fpkm()' in DESeq2 using the combined exon length as gene length. The mean FPKM value between replicates was used for downstream analyses. 


\section{Image analysis}

Image analysis was performed with custom ImageJ 2.0.0 and R scripts.

To calculate ${ }^{\mathrm{m} 6} \mathrm{~A}$-Tracer enrichment around nucleoli, individual nuclei were first segmented using the DAPI signal. This was done using a sequence of noise filtering (Gaussian blur, radius of 2 pixels), contrast enhancement ( $0.2 \%$ saturated pixels) and segmentation (Otsu method), after which a watershed algorithm was used to separate closely positioned nuclei. Nuclei positioned at image borders and with abnormal dimensions (due to faulty segmentation) were removed. Ki67 antibody signal was noise-filtered (Gaussian blur, radius of 2 pixels), background-subtracted (rolling ball radius of 500 pixels) and contrast enhanced up to $0.1 \%$ saturated pixels. These images were then used to segment Ki67 domains (that we interpret as nucleoli) using a fixed threshold of 180 (of 255). Any holes were filled. The segmented domains were used to calculate distance masks from and into Ki67 domains. For every nucleus, distance maps were overlayed with blurred and background subtracted signals for ${ }^{\mathrm{m} 6} \mathrm{~A}-\mathrm{Tracer}$ and Ki67 antibody signals, and per distance group a $\log _{2}$-ratio was calculated over the average nuclear intensity. Finally, nuclei were filtered to have reasonable enrichment scores, which removes cells with faulty segmentation and mitotic cells. Average signal traces were shown for individual nuclei, and averaged between all nuclei of a particular experimental condition.

To calculate Ki67 depletion efficiency in HCT116 Ki67-AID cells for the two additional Ki67 antibodies (Novus and HPA), individual nuclei were segmented as described above. Ki67 antibody and mClover signals were noise-filtered (Gaussian blur, radius of 2 pixels), median-smoothed (radius of 3 pixels) and background subtracted (rolling window of 250 pixels). Average signals were calculated for every nucleus. For the initial Ki67 antibody (Abcam), 3D stacks were imaged rather than sections through the middle plane. Image analysis was performed as for the 2D slices, but using 3D implementations instead. 3D Gaussian blurring was performed with a radius of 1 pixel in every direction.

\section{Centromere definition}

Locations of centromeres were downloaded from the UCSC table browser (group: mapping and sequencing; track: centromeres).

\section{External data}

Supplementary Table 1 lists the external data sets that have been used in this study. Previously generated pA-DamID reads were used from the 4DN data repository (https://data.4dnucleome.org/) and processed as described above. These data sets are included in the processed data files that can be found in the GEO repository linked below. Processed Repli-seq data in $5 \mathrm{~kb}$ bins was downloaded from the 4DN data repository and the average score was calculated for $50 \mathrm{~kb}$ bins as described above. RNA-seq reads were downloaded from multiple references and used to calculate average FPKM values as described above. 
535

536

537

538

539

540

541

542

543

544

545

546

547

548

549

550

551

552

553

554

555

556

557

558

559

560

561

562

563

564

\section{Data availability}

Data sets, computational code and lab journal entries that have been produced for this study are available in the following repositories:

- Data generated in this manuscript: GEO data repository (GSE186206)

- Computer scripts: will be made available

- Lab journal records: will be made available

\section{ACKNOWLEDGEMENTS}

We thank Masatoshi Takagi for the HCT116 Ki67-AID cell line, and the NKI Digital Microscopy, Flow Cytometry, Genomics, Protein Production, and RHPC core facilities for technical assistance. We thank members of the BvS lab; Andrew Belmont and other members of the 4D Nucleome Center for Nuclear Cytomics for helpful suggestions. Supported by NIH Common Fund "4D Nucleome" Program grant U54 DK107965 (BvS, DMG), ERC Advanced Grant 694466 (BvS), MSCA/AIRC iCARE2.0 fellowship 800924 (SGM), and Marie Curie Fellowship 838555 (SGM). The Oncode Institute is partly supported by KWF Dutch Cancer Society.

\section{AUTHOR CONTRIBUTIONS}

TvS: Conceived and designed study, conducted majority of experiments and all data analysis, wrote manuscript. SGM: Performed experiments. AV: Performed Repli-seq experiments. DMG: Supervised project. BvS: Designed study, wrote manuscript, supervised project.

\section{CONFLICT OF INTEREST}

The authors declare no conflicting interests. 


\section{REFERENCES}

1. Scholzen, T. and J. Gerdes, The Ki-67 protein: from the known and the unknown. J Cell Physiol, 2000. 182(3): p. 311-22.

2. Sun, X. and P.D. Kaufman, Ki-67: more than a proliferation marker. Chromosoma, 2018. 127(2): p. 175-186.

3. Remnant, L., et al., The intrinsically disorderly story of Ki-67. Open Biol, 2021. 11(8): p. 210120.

4. Verheijen, R., et al., Ki-67 detects a nuclear matrix-associated proliferation-related antigen. II. Localization in mitotic cells and association with chromosomes. J Cell Sci, 1989. 92 ( Pt 4): p. 531-40.

5. Booth, D.G., et al., Ki-67 is a PP1-interacting protein that organises the mitotic chromosome periphery. Elife, 2014. 3: p. e01641.

6. Cuylen, S., et al., Ki-67 acts as a biological surfactant to disperse mitotic chromosomes. Nature, 2016. 535(7611): p. 308-12.

7. Takagi, M., et al., Perichromosomal protein Ki67 supports mitotic chromosome architecture. Genes Cells, 2016. 21(10): p. 1113-1124.

8. Cuylen-Haering, S., et al., Chromosome clustering by Ki-67 excludes cytoplasm during nuclear assembly. Nature, 2020. 587(7833): p. 285-290.

9. Ochs, R.L., et al., Nucleologenesis: composition and fate of prenucleolar bodies. Chromosoma, 1985. 92(5): p. 330-6.

10. Dundr, M., T. Misteli, and M.O. Olson, The dynamics of postmitotic reassembly of the nucleolus. J Cell Biol, 2000. 150(3): p. 433-46.

11. Savino, T.M., et al., Nucleolar assembly of the rRNA processing machinery in living cells. J Cell Biol, 2001. 153(5): p. 1097-110.

12. Carron, C., et al., Post-mitotic dynamics of pre-nucleolar bodies is driven by prerRNA processing. J Cell Sci, 2012. 125(Pt 19): p. 4532-42.

13. Ohno, S., W.D. Kaplan, and R. Kinosita, The centromeric and nucleolus-associated heterochromatin of Rattus norvegicus. Exp Cell Res, 1959. 16(2): p. 348-57.

14. Lima-De-Faria, A. and J. Reitalu, Heterochromatin in human male leukocytes. J Cell Biol, 1963. 16: p. 315-22.

15. Guelen, L., et al., Domain organization of human chromosomes revealed by mapping of nuclear lamina interactions. Nature, 2008. 453(7197): p. 948-51.

16. van Koningsbruggen, S., et al., High-resolution whole-genome sequencing reveals that specific chromatin domains from most human chromosomes associate with nucleoli. Mol Biol Cell, 2010. 21(21): p. 3735-48.

17. Dillinger, S., T. Straub, and A. Nemeth, Nucleolus association of chromosomal domains is largely maintained in cellular senescence despite massive nuclear reorganisation. PLoS One, 2017. 12(6): p. e0178821.

18. Vertii, A., et al., Two contrasting classes of nucleolus-associated domains in mouse fibroblast heterochromatin. Genome Res, 2019. 29(8): p. 1235-1249.

19. Kind, J., et al., Single-cell dynamics of genome-nuclear lamina interactions. Cell, 2013. 153(1): p. 178-92.

20. Ragoczy, T., et al., Functional redundancy in the nuclear compartmentalization of the late-replicating genome. Nucleus, 2014. 5(6): p. 626-35.

21. Solovei, I., et al., $L B R$ and lamin $A / C$ sequentially tether peripheral heterochromatin and inversely regulate differentiation. Cell, 2013. 152(3): p. 584-98.

22. Sobecki, M., et al., The cell proliferation antigen Ki-67 organises heterochromatin. Elife, 2016. 5: p. e13722.

23. Matheson, T.D. and P.D. Kaufman, The $p 150 \mathrm{~N}$ domain of chromatin assembly factor1 regulates Ki-67 accumulation on the mitotic perichromosomal layer. Mol Biol Cell, 2017. 28(1): p. 21-29.

24. van Schaik, T., et al., Cell cycle dynamics of lamina-associated DNA. EMBO Rep, 2020. 21(11): p. e50636. 
25. Greil, F., C. Moorman, and B. van Steensel, DamID: mapping of in vivo proteingenome interactions using tethered DNA adenine methyltransferase. Methods Enzymol, 2006. 410: p. 342-59.

26. Bolzer, A., et al., Three-dimensional maps of all chromosomes in human male fibroblast nuclei and prometaphase rosettes. PLoS Biol, 2005. 3(5): p. e157.

27. Su, J.H., et al., Genome-Scale Imaging of the $3 D$ Organization and Transcriptional Activity of Chromatin. Cell, 2020. 182(6): p. 1641-1659 e26.

28. Nemeth, A. and I. Grummt, Dynamic regulation of nucleolar architecture. Curr Opin Cell Biol, 2018. 52: p. 105-111.

29. Zhang, L.F., K.D. Huynh, and J.T. Lee, Perinucleolar targeting of the inactive $X$ during $S$ phase: evidence for a role in the maintenance of silencing. Cell, 2007. 129(4): p. 693-706.

30. Sun, $\mathrm{X}$., et al., Ki-67 Contributes to Normal Cell Cycle Progression and Inactive $X$ Heterochromatin in p21 Checkpoint-Proficient Human Cells. Mol Cell Biol, 2017. 37(17).

31. Zatsepina, O.V., et al., Experimental induction of prenucleolar bodies (PNBs) in interphase cells: interphase PNBs show similar characteristics as those typically observed at telophase of mitosis in untreated cells. Chromosoma, 1997. 105(7-8): p. 418-30.

32. Perry, R.P. and D.E. Kelley, Inhibition of RNA synthesis by actinomycin D: characteristic dose-response of different RNA species. J Cell Physiol, 1970. 76(2): p. 127-39.

33. Politz, J.C.R., D. Scalzo, and M. Groudine, The redundancy of the mammalian heterochromatic compartment. Curr Opin Genet Dev, 2016. 37: p. 1-8.

34. Bizhanova, A. and P.D. Kaufman, Close to the edge: Heterochromatin at the nucleolar and nuclear peripheries. Biochim Biophys Acta Gene Regul Mech, 2021. 1864(1): p. 194666.

35. Marchal, C., J. Sima, and D.M. Gilbert, Control of DNA replication timing in the $3 D$ genome. Nat Rev Mol Cell Biol, 2019. 20(12): p. 721-737.

36. Dekker, J., et al., The 4D nucleome project. Nature, 2017. 549(7671): p. 219-226.

37. Marchal, C., et al., Genome-wide analysis of replication timing by next-generation sequencing with E/L Repli-seq. Nat Protoc, 2018. 13(5): p. 819-839.

38. Reddy, K.L., et al., Transcriptional repression mediated by repositioning of genes to the nuclear lamina. Nature, 2008. 452(7184): p. 243-7.

39. Hiratani, I., et al., Global reorganization of replication domains during embryonic stem cell differentiation. PLoS Biol, 2008. 6(10): p. e245.

40. van Steensel, B. and A.S. Belmont, Lamina-Associated Domains: Links with Chromosome Architecture, Heterochromatin, and Gene Repression. Cell, 2017. 169(5): p. 780-791.

41. Mrouj, K., et al., Ki-67 regulates global gene expression and promotes sequential stages of carcinogenesis. Proc Natl Acad Sci U S A, 2021. 118(10).

42. Garwain, O., et al., The chromatin-binding domain of Ki-67 together with p53 protects human chromosomes from mitotic damage. bioRxiv, 2020: p. 2020.10.16.342352.

43. Gerdes, J., et al., Cell cycle analysis of a cell proliferation-associated human nuclear antigen defined by the monoclonal antibody Ki-67. J Immunol, 1984. 133(4): p. 17105.

44. Stenstrom, L., et al., Mapping the nucleolar proteome reveals a spatiotemporal organization related to intrinsic protein disorder. Mol Syst Biol, 2020. 16(8): p. e9469.

45. Saiwaki, T., et al., In vivo dynamics and kinetics of pKi-67: transition from a mobile to an immobile form at the onset of anaphase. Exp Cell Res, 2005. 308(1): p. 123-34.

46. Crabbe, L., et al., Human telomeres are tethered to the nuclear envelope during postmitotic nuclear assembly. Cell Rep, 2012. 2(6): p. 1521-9.

47. Dechat, T., et al., LAP2alpha and BAF transiently localize to telomeres and specific regions on chromatin during nuclear assembly. J Cell Sci, 2004. 117(Pt 25): p. 611728. 
674

675

676

677

678

679

680

681

682

683

684

685

686

687

688

689

690

691

692

693

694

695

696

697

698

699

700

701

702

703

704

705

706

707

708

709

710

711

712

713

714

715

716

717

718

719

720

721

722

723

724

48. MacCallum, D.E. and P.A. Hall, Biochemical characterization of pKi67 with the identification of a mitotic-specific form associated with hyperphosphorylation and altered DNA binding. Exp Cell Res, 1999. 252(1): p. 186-98.

49. Bridger, J.M., I.R. Kill, and P. Lichter, Association of pKi-67 with satellite DNA of the human genome in early G1 cells. Chromosome Res, 1998. 6(1): p. 13-24.

50. Quinodoz, S.A., et al., Higher-Order Inter-chromosomal Hubs Shape 3D Genome Organization in the Nucleus. Cell, 2018. 174(3): p. 744-757 e24.

51. MacCallum, D.E. and P.A. Hall, The biochemical characterization of the DNA binding activity of pKi67. J Pathol, 2000. 191(3): p. 286-98.

52. Luderus, M.E., et al., Binding of matrix attachment regions to lamin polymers involves single-stranded regions and the minor groove. Mol Cell Biol, 1994. 14(9): p. 6297305.

53. Meuleman, W., et al., Constitutive nuclear lamina-genome interactions are highly conserved and associated with A/T-rich sequence. Genome Res, 2013. 23(2): p. 270-80.

54. Sobecki, M., et al., Cell-Cycle Regulation Accounts for Variability in Ki-67 Expression Levels. Cancer Res, 2017. 77(10): p. 2722-2734.

55. Miller, I., et al., Ki67 is a Graded Rather than a Binary Marker of Proliferation versus Quiescence. Cell Rep, 2018. 24(5): p. 1105-1112 e5.

56. Bloom, K. and V. Costanzo, Centromere Structure and Function. Prog Mol Subcell Biol, 2017. 56: p. 515-539.

57. Barra, V. and D. Fachinetti, The dark side of centromeres: types, causes and consequences of structural abnormalities implicating centromeric DNA. Nat Commun, 2018. 9(1): p. 4340.

58. Bury, L., et al., Alpha-satellite RNA transcripts are repressed by centromerenucleolus associations. Elife, 2020. 9.

59. Giunta, S., et al., CENP-A chromatin prevents replication stress at centromeres to avoid structural aneuploidy. Proc Natl Acad Sci U S A, 2021. 118(10).

60. Vogel, M.J., D. Peric-Hupkes, and B. van Steensel, Detection of in vivo protein-DNA interactions using DamID in mammalian cells. Nat Protoc, 2007. 2(6): p. 1467-78.

61. Leemans, C., et al., Promoter-Intrinsic and Local Chromatin Features Determine Gene Repression in LADs. Cell, 2019. 177(4): p. 852-864 e14.

62. Chen, S., et al., fastp: an ultra-fast all-in-one FASTQ preprocessor. Bioinformatics, 2018. 34(17): p. i884-i890.

63. Dobin, A., et al., STAR: ultrafast universal RNA-seq aligner. Bioinformatics, 2013. 29(1): p. 15-21.

64. Love, M.I., W. Huber, and S. Anders, Moderated estimation of fold change and dispersion for RNA-seq data with DESeq2. Genome Biol, 2014. 15(12): p. 550.

65. Consortium, E.P., An integrated encyclopedia of DNA elements in the human genome. Nature, 2012. 489(7414): p. 57-74.

66. Kelso, T.W.R., et al., Chromatin accessibility underlies synthetic lethality of SWI/SNF subunits in ARID1A-mutant cancers. Elife, 2017. 6.

67. Dai, Z., et al., Methionine metabolism influences genomic architecture and gene expression through H3K4me3 peak width. Nat Commun, 2018. 9(1): p. 1955.

68. Slaats, G.G., et al., Screen-based identification and validation of four new ion channels as regulators of renal ciliogenesis. J Cell Sci, 2015. 128(24): p. 4550-9.

69. Durrbaum, M., et al., The deregulated microRNAome contributes to the cellular response to aneuploidy. BMC Genomics, 2018. 19(1): p. 197.

70. Harenza, J.L., et al., Transcriptomic profiling of 39 commonly-used neuroblastoma cell lines. Sci Data, 2017. 4: p. 170033. 


\section{FIGURE LEGENDS} DamID

(A) Schematic overview of pA-DamID [24]. Permeabilized cells are incubated with a primary antibody (e.g. against Ki67), followed by a fusion of proteinA and Dam (pA-Dam). After removal of unbound pA-Dam, the Dam enzyme is activated by addition of Sadenosylmethionine (SAM), resulting in local deposition of ${ }^{\mathrm{m} 6} \mathrm{~A}$ marks. ${ }^{\mathrm{m} 6} \mathrm{~A}$-marked DNA can be processed for high-throughput sequencing, or alternatively cells can be fixed and ${ }^{m 6} A$ marks visualized using the ${ }^{\mathrm{m} 6} \mathrm{~A}-$ Tracer protein.

(B) Representative confocal microscopy sections of HCT116 cells following pA-DamID with free Dam (top panel) or Ki67 antibody (bottom panel), labeled with ${ }^{\mathrm{m} 6} \mathrm{~A}-\mathrm{Tracer}$ protein and stained for Ki67. Scale bar: $5 \mu \mathrm{m}$.

(C) Quantification of the enrichment of Ki67 antibody and ${ }^{\mathrm{m} 6} \mathrm{~A}-$ Tracer signals relative to segmented Ki67 domains (that we interpret as nucleoli) in different cell lines. For every cell, the enrichment is calculated by pixel-distances relative to the mean signal of that cell, and represented as a $\log _{2}$-ratio. Negative distances are outside of Ki67 domains, a distance of zero marks the domain boundary and positive distances are inside of Ki67 domains. Every thin line corresponds to an individual cell and the thick line is the mean of all cells. Results are combined from three (hTERT-RPE) or one (HCT116 and K562) biological replicates. (D) Comparison of Ki67 pA-DamID profiles ( $\log _{2}$-ratios over the free Dam control) across two chromosomes in hTERT-RPE, HCT116 and K562 cells. Sequenced reads are counted and normalized in $50 \mathrm{~kb}$ bins. Data are averages of $n$ biological replicates and smoothed with a running mean across nine $50 \mathrm{~kb}$ bins for visualization purposes. Centromeres are highlighted by black bars.

751

(E) Distributions of Ki67 interactions for all chromosomes, ordered by decreasing chromosome size. rDNA-containing chromosomes are highlighted by black borders. Boxplots: horizontal lines represent $25^{\text {th }}, 50^{\text {th }}$, and $75^{\text {th }}$ percentiles; whiskers extend to $5^{\text {th }}$ and $95^{\text {th }}$ percentiles.

754 (F) Distributions of Ki67 interactions nearby centromeres. Boxplots are drawn for every 0.5 $\mathrm{Mb}$, following the specification as in $(\mathrm{E})$ with the $50^{\text {th }}$ percentile highlighted in red. (G) The mean Ki67 interaction score near centromeres is plotted for each chromosome ordered by size (within $2 \mathrm{Mb}$ of centromeres, overlapping the enrichment in $(\mathrm{F})$ ). rDNAcontaining chromosomes are highlighted in red.

(A) Representative confocal microscopy sections of hTERT-RPE cells processed with Ki67 pA-DamID, following synchronization with a mitotic shake-off ( $t=0 \mathrm{~h}$, see Methods) and time points after replating of the synchronized cells. Cells were stained for Ki67 and labeled with 
Instead of individual cell traces, the $95 \%$-confidence interval of the mean is added as shaded area. Results are combined from three (unsynchronized cells) or one (synchronized cells) biological replicates.

770 (C) Ki67 interactions profiles for the synchronized hTERT-RPE cells. Two representative chromosomes were visualized to highlight the differences between large (chr2) and small chromosomes (chr22). Log $_{2}$-ratios were converted to z-scores to correct for differences in dynamic range between conditions and replicates. Data profiles are averages of $n$ experiments and smoothed with a running mean across nine $50 \mathrm{~kb}$ bins. Centromeres are highlighted by black bars.

(D) Smooth loess curves of Ki67 interactions plotted against the distance to chromosome ends for cells synchronized in metaphase $(t=0 \mathrm{~h})$. Lines represent individual chromosomes. (E) Chromosomal distributions of genome-wide differences between Ki67 interactions in early $\mathrm{G} 1(\mathrm{t}=1 \mathrm{~h}$ ) and unsynchronized cells. Boxplots: horizontal lines represent 25th, 50th, and 75 th percentiles; whiskers extend to 5 th and 95th percentiles. (F) Mean chromosomal differences in Ki67 interaction scores between interphase time points and early $\mathrm{G} 1$ cells $(\mathrm{t}=1 \mathrm{~h}$ ) are plotted for chromosomes sorted by size.

Fig 3. Ki67 interactions are restricted by nucleolar positioning

(A) Representative confocal microscopy sections of hTERT-RPE cells following 3 hours of a low dose of actinomycin D (ActD, $50 \mathrm{ng} / \mathrm{mL}$ in $0.05 \%$ dimethyl sulfoxide (DMSO)) to disrupt nucleoli, and of cells treated with a similar quantity of DMSO as control. Cells were stained for MKI67IP and Ki67 proteins to visualize nucleolar disruption and new Ki67 localization patterns. Scale bar: $10 \mu \mathrm{m}$.

(B) Comparison of Ki67 interactions in the three cell lines after ActD-induced nucleolar disruption for two representative chromosomes. DMSO control and ActD treated cells are shown in red and blue, respectively. Data are averages of two experiments and smoothed with a running mean across nine $50 \mathrm{~kb}$ bins for visualization. Centromeres are highlighted by black bars.

(C) The differences between ActD and control conditions in mean chromosomal Ki67 signal is plotted for chromosomes sorted by size. rDNA-containing chromosomes are highlighted with triangles.

798 (D) The difference between ActD and control conditions in mean Ki67 interactions scores 799 near centromeres is plotted for each chromosome (within 2Mb of centromeres, overlapping the enrichment in (Fig 1F) ). rDNA-containing chromosomes are highlighted in red.

803 (A) Comparison of Ki67 interactions with Lamin B1 interactions (both $\log _{2}$-ratios) and DNA 804 replication timing (Repli-seq $\log _{2}$-ratio of Early/Late) for a representative genomic region. 805 Orange arrows highlight domains with high Ki67 and generally low Lamin B1 interactions, 806 and vice-versa. Data profiles are averages of $n$ experiments and smoothed with a running mean across nine $50 \mathrm{~kb}$ bins. Centromeres are highlighted by black bars. 
808 (B) Binned scatterplots between Ki67 and Lamin B1 interactions ( $\log _{2}$-ratios), showing the

809 amount of genomic $50 \mathrm{~kb}$ bins overlapping these points (top panels) and the mean replication

810 timing score of overlapping genomic bins ( $\log _{2}$ Early/Late) (bottom panels). Points are only

811 shown with at least 10 overlapping genomic bins for a robust estimation of mean scores. A

812 separate color bar is used for replication timing in K562 cells, which have a reduced dynamic

813 range. Replication timing data were obtained from the 4D Nucleome data repository [36].

814

815 Fig 5. Effects of Ki67 depletion on Lamin B1 interactions and replication timing

816 (A) Overview of the Ki67 depletion experiment in HCT116 Ki67-AID cells (top). Cells were

817 either cultured with a double thymidine arrest to enrich for S-phase cells (which includes one 818 cell division) or arrested in G2 by CDK1 inhibition immediately following an initial S-phase 819 arrest (which prevents a cell division). FACS analysis confirmed successful synchronization 820 and illustrates that Ki67 depletion does not affect this (bottom panels).

821 (B) Differences in mean Ki67 chromosome scores between S- and G2-synchronized 822 HCT116 Ki67-AID cells.

823 (C) Representative genomic region showing the effect of Ki67 depletion on Lamin B1 824 interactions, for HCT116 Ki67-AID cells synchronized in S-phase (left panel) and G2-phase

825 (right panel). Black and red lines represent conditions with normal and depleted Ki67 levels,

826 respectively. Ki67 interactions were only profiled at non-depleted levels. Orange arrows

827 highlight regions with high Ki67 interactions that gain Lamin B1 interactions upon Ki67

828 depletion. Data profiles are averages of 2 experiments and smoothed with a running mean

829 across nine $50 \mathrm{~kb}$ bins. The centromere is located just to the right of the depicted region.

830 (D) Binned scatterplots of Lamin B1 interactions at in HCT116 cells with normal versus

831 depleted Ki67 levels, as described in (Fig 4B). Bins are colored by mean Ki67 interactions in

832 S-phase (top panels) and G2-phase (bottom panels). Signals are smoothed with a running

833 mean of three $50 \mathrm{~kb}$ bins to reduce noise in the data.

834 (E) Representative genomic region showing the effect of auxin-mediated Ki67 depletion on

835 (S-phase) Lamin B1 interactions and replication timing ( $\log _{2}$ Early/Late) in HCT116 Ki67-AID

836 cells, as described in (C). Orange arrows highlight domains with gained Lamin B1

837 interactions and delayed replication timing; purple arrows highlight domains with gained

838 Lamin B1 interactions but unaffected replication timing.

839 (F) Binned scatterplots (as in (D)) of replication timing (RT, E/L) in cells with normal versus

840 depleted Ki67 levels, colored by the density of overlapping $50 \mathrm{~kb}$ bins (left panel) and mean

841 Ki67 interactions at normal Ki67 levels (right panel).

842 (G) Distribution of differences in replication timing (RT) nearby centromeres. Boxplots:

843 horizontal lines represent 25th, 50th, and 75th percentiles; whiskers extend to 5th and 95th 844 percentiles.

845 (H) Mean difference in replication timing (RT) within $2 \mathrm{Mb}$ from the centromere of each 846 chromosome. rDNA-containing chromosomes are highlighted in red. 
848 Fig 6. Comparison of the Ki67 and Lamin B1 balance with H3K27me3 and H3K9me3

849 (A) Comparison of Ki67 interactions with Lamin B1 interactions and H3K27me3 and $850 \mathrm{H} 3 \mathrm{~K} 9 \mathrm{me} 3$ histone modifications for a representative genomic region. Data profiles are 851 averages of $n$ pA-DamID experiments and smoothed with a running mean across $950 \mathrm{~kb}$ 852 bins. Centromeres are highlighted by black bars.

853 (B) Binned scatterplots of Ki67 and Lamin B1 interactions as in (Fig 4B), colored according 854 to the mean $\mathrm{H} 3 \mathrm{~K} 27$ me3 and $\mathrm{H} 3 \mathrm{~K} 9 \mathrm{me} 3$ signals.

855 (C) A similar figure as (Fig 5B), but additionally showing the effect of Ki67 depletion on 856 H3K27me3 and H3K9me3.

857 (D) Similar plots as (Fig 5D), but instead showing H3K27me3 and H3K9me3 at normal and 858 depleted Ki67 levels. Bins are colored by the Ki67 interactions in cells with normal Ki67 859 levels.

860

861

862

\section{SUPPLEMENTARY FIGURE LEGENDS}

863

Fig S1. Additional information and controls for Ki67 pA-DamID

865

(A) Example of raw Ki67 pA-DamID profiles for a representative experiment in HCT116 cells.

${ }^{\mathrm{m} 6} \mathrm{~A}$-marked DNA is specifically amplified, sequenced, counted in $50 \mathrm{~kb}$ bins and counts-per-

867 million normalized (cpm) for Ki67 (top profile) and free Dam (middle profile). A $\log _{2}$-ratio between Ki67 and Dam accounts for DNA accessibility and amplification bias, and is used as a measure of Ki67 interactions. Data profiles are smoothed with a running mean across nine $50 \mathrm{~kb}$ bins for visualization purposes. Centromeres are highlighted by red bars.

871

(B) Replicate experiment correlations for Ki67 interactions ( $\log _{2}$-ratios) measured in

872 unsynchronized HCT116 cells (top panel) and K562 cells (bottom panel).

873 (C) Overview of Pearson correlations between replicate experiments for all experimental

874 conditions studied throughout this manuscript, separated by cell line and including

875 perturbation experiments.

876 (D) Representative maximum projections of confocal microscopy stacks of HCT116 Ki67-

877 miniAID-mClover cells (HCT116 Ki67-AID in short) with normal (top panels) and auxin-

878 mediated depleted Ki67 levels (bottom panels). Ki67 levels were visualized directly by

879 mClover fluorescence, and indirectly by immunofluorescence with a Ki67 antibody. Scale 880 bar: $10 \mu \mathrm{m}$.

881 (E) Quantification of Ki67 levels measured by mClover (middle panel) and immunostaining

882 (bottom panel) from three independent replicates. Every point represents a single cell.

883 (F) Two representative chromosomes showing the effect of auxin-mediated Ki67 depletion in 884 HCT116 Ki67-AID cells on Ki67 interactions as measured by pA-DamID. Data are averages 885 of two biological replicates and smoothed with a running mean across nine $50 \mathrm{~kb}$ bins.

886 Centromeres are marked by red bars.

887 (G) Correlation of all $50 \mathrm{~kb}$ genomic bins from profiles described in $(F)$. 

immunostaining signals in HCT116 Ki67-AID cells using different Ki67 antibodies. For this experiment, single confocal microscopy sections from the middle of cell nuclei were used rather than maximum projections from entire nuclei. Results are combined from two biological replicates.

(B) Quantification of ${ }^{\mathrm{m} 6} \mathrm{~A}-\mathrm{Tracer}$ enrichment around Ki67-marked nucleoli, as described in (Fig 1B-C). Results are combined from one (middle-targeting antibody) or two ( $\mathrm{N}$ - and $\mathrm{C}$ terminus antibodies) biological replicates. Data from Fig $1 \mathrm{C}$ are not included here.

(C) Ki67 interactions on two representative chromosomes as determined by pA-DamID using three different Ki67 antibodies. Data profiles are averages of $n$ biological replicates and smoothed with a running mean across nine $50 \mathrm{~kb}$ bins. Centromeres are highlighted by black bars.

902

(D-E) Correlation of all $50 \mathrm{~kb}$ genomic bins between Ki67 interactions profiled with the middle-targeting antibody and the $\mathrm{N}-(\mathrm{D})$ and $\mathrm{C}$-terminus antibodies (E).

Fig S3. Ki67 interactions are more conserved on small chromosomes, and are not enriched at rDNA repeats

(A) Pearson correlations between cell lines along all $50 \mathrm{~kb}$ genomic bins of individual chromosomes. The panel title indicates the first cell line and the color indicates the second cell line between which the correlation was calculated.

910 (B) Enrichment of rDNA sequences detected by Ki67 pA-DamID compared to the Dam control.

Fig S4. A short osmotic shock recapitulates Ki67 maturation from PNBs to nucleoli (A) Representative confocal microscopy sections of hTERT-RPE cells before a 15-minute osmotic shock (0.2x PBS) (top panels), and after 30 and 180 minutes of recovery in complete medium (middle and bottom panels). Formaldehyde-fixed cells were stained for MKI67IP and Ki67. Scale bar: $10 \mu \mathrm{m}$.

918 (B) Representative chromosomes showing Ki67 interactions in unsynchronized hTERT-RPE 919 cells, two interphase time points (data are the same as in Fig 2C) and the two time points following osmotic shock. Note that the regions that change Ki67 interactions during interphase show the same trends after the osmotic shock. Data profiles are averages of two experiments and smoothed with a running mean across nine $50 \mathrm{~kb}$ bins. Centromeres are highlighted by black bars.

924 (C) Changes in chromosomal distributions of Ki67 are similar for progression in $\mathrm{G} 1$ phase ( $\mathrm{x}$ 925 axis: difference in mean interactions per chromosome between $10 \mathrm{~h}$ and $1 \mathrm{~h}$ after mitosis) and 926 upon recovery from osmotic shock (y-axis: difference in mean interactions per chromosome 927 between $180 \mathrm{~min}$ and $30 \mathrm{~min}$ after termination of the osmotic shock (OS)).

928 (D-E) Representative confocal microscopy sections showing the effect of ActD on nucleolar morphology in HCT116 (D) and K562 cells (E), as described in (Fig 3A). Scale bar: $10 \mu \mathrm{m}$. 
931 Fig S5. Loss of Ki67 interactions does not correlate with gene expression changes

932 (A) Bar plot showing average replication timing ( $\log _{2}$-ratio Early/Late) for centromeres. All

933 reads were selected that aligned to regions marked as centromeres (UCSC Table browser;

934 hg38 centromeres) regardless of their mapping quality and, following counts-per-million

935 normalization, the E/L ratio was calculated between the total centromeric counts. Between 3-

$9365 \%$ of the late-fraction mapped to centromeres.

937 (B-C) Similar plots as (Fig 5G-H), but showing differences in Lamin B1 interactions instead.

938 (D) Similar plot as (Fig 4B), but instead highlighting for every gene the expression level (in

$939 \log _{10}$ FPKM). Expression data are used from [30, 65-70] (see Methods).

940 (E) Scatterplot of mean gene expression (counts per million; cpm) versus expression

941 difference ( $\log _{2}$-fold change) following auxin-mediated Ki67 depletion in HCT116 Ki67-AID

942 cells. Significant genes (Benjamini-Hochberg adjusted $p$-value $<0.05$ ) are highlighted in red.

943 (F) Boxplots showing Ki67 interactions for differentially expressed genes following Ki67

944 depletion in hTERT-RPE cells. Gene expression calls are from [30]. Boxplots: horizontal

945 lines represent 25th, 50th, and 75th percentiles; whiskers extend to 5th and 95th percentiles.

946 


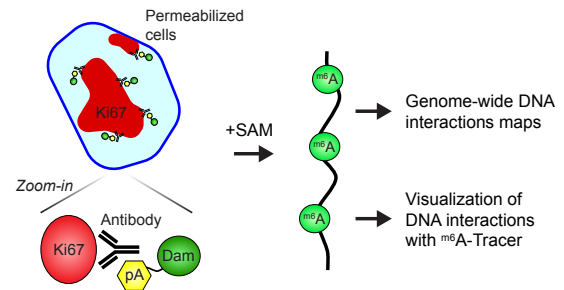

B

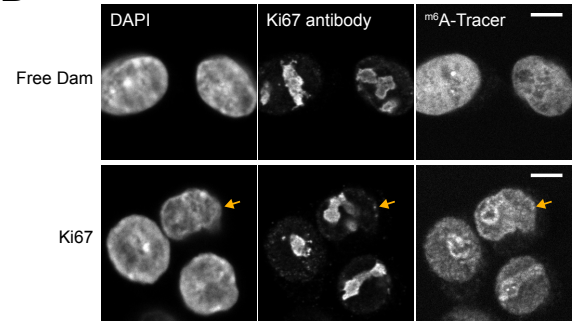

C

व

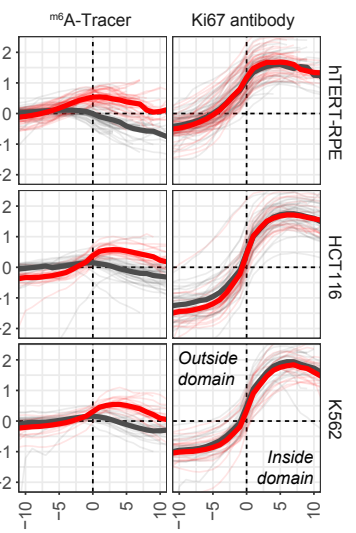

pA-DamID target - Free Dam - Ki67

Distance to Ki67 domain (pixel)

D
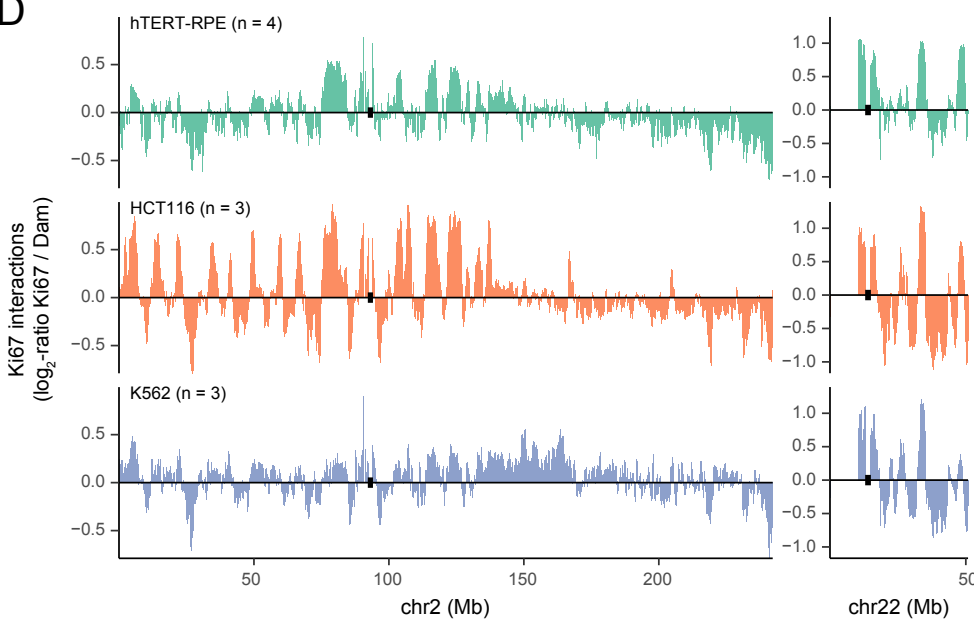

$E$

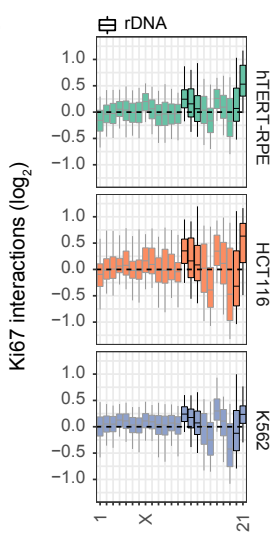

Chromosomes (by size)
F

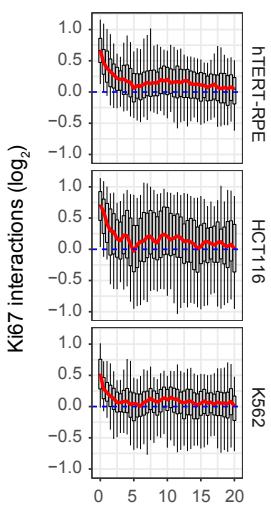

Distance to centromere $(\mathrm{Mb})$

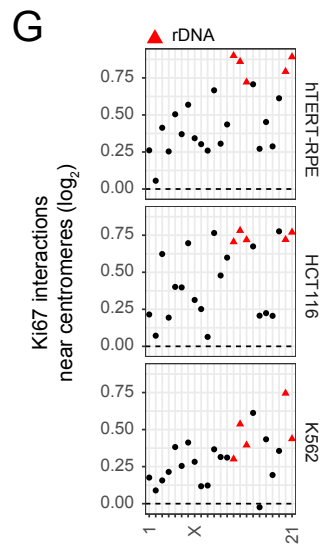

Chromosomes (by size)

\section{Fig 1. Visualization and genome-wide profiling of DNA-Ki67 interactions using pA-DamID}

(A) Schematic overview of pA-DamID [24]. Permeabilized cells are incubated with a primary antibody (e.g. against Ki67), followed by a fusion of proteinA and Dam (pA-Dam). After removal of unbound pA-Dam, the Dam enzyme is activated by addition of S-adenosylmethionine (SAM), resulting in local deposition of m6A marks. m6A-marked DNA can be processed for high-throughput sequencing, or alternatively cells can be fixed and m6A marks visualized using the m6A-Tracer protein.

(B) Representative confocal microscopy sections of HCT116 cells following pA-DamID with free Dam (top panel) or Ki67 antibody (bottom panel), labeled with m6A-Tracer protein and stained for Ki67. Scale bar: $5 \mu \mathrm{m}$.

(C) Quantification of the enrichment of Ki67 antibody and m6A-Tracer signals relative to segmented Ki67 domains (that we interpret as nucleoli) in different cell lines. For every cell, the enrichment is calculated by pixel-distances relative to the mean signal of that cell, and represented as a log2-ratio. Negative distances are outside of Ki67 domains, a distance of zero marks the domain boundary and positive distances are inside of Ki67 domains. Every thin line corresponds to an individual cell and the thick line is the mean of all cells. Results are combined from three (hTERT-RPE) or one (HCT116 and K562) biological replicates.

(D) Comparison of Ki67 pA-DamID profiles (log2-ratios over the free Dam control) across two chromosomes in hTERT-RPE, HCT116 and K562 cells. Sequenced reads are counted and normalized in $50 \mathrm{~kb}$ bins. Data are averages of $\mathrm{n}$ biological replicates and smoothed with a running mean across nine $50 \mathrm{~kb}$ bins for visualization purposes. Centromeres are highlighted by black bars.

(E) Distributions of Ki67 interactions for all chromosomes, ordered by decreasing chromosome size. rDNA-containing chromosomes are highlighted by black borders. Boxplots: horizontal lines represent 25th, 50th, and 75th percentiles; whiskers extend to 5th and 95th percentiles.

(F) Distributions of Ki67 interactions nearby centromeres. Boxplots are drawn for every $0.5 \mathrm{Mb}$, following the specification as in (E) with the 50 th percentile highlighted in red.

(G) The mean Ki67 interaction score near centromeres is plotted for each chromosome ordered by size (within 2Mb of centromeres, overlapping the enrichment in ( $F$ )). rDNA-containing chromosomes are highlighted in red. 
$A$

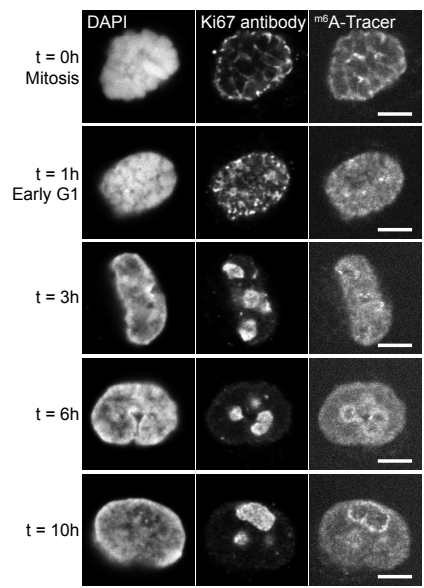

B

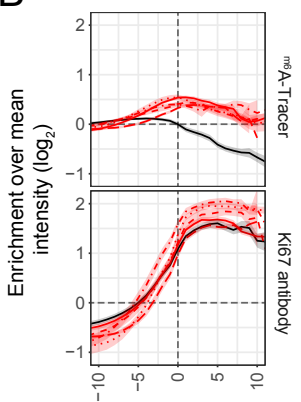

Distance to Ki67 domains (pixel)

C
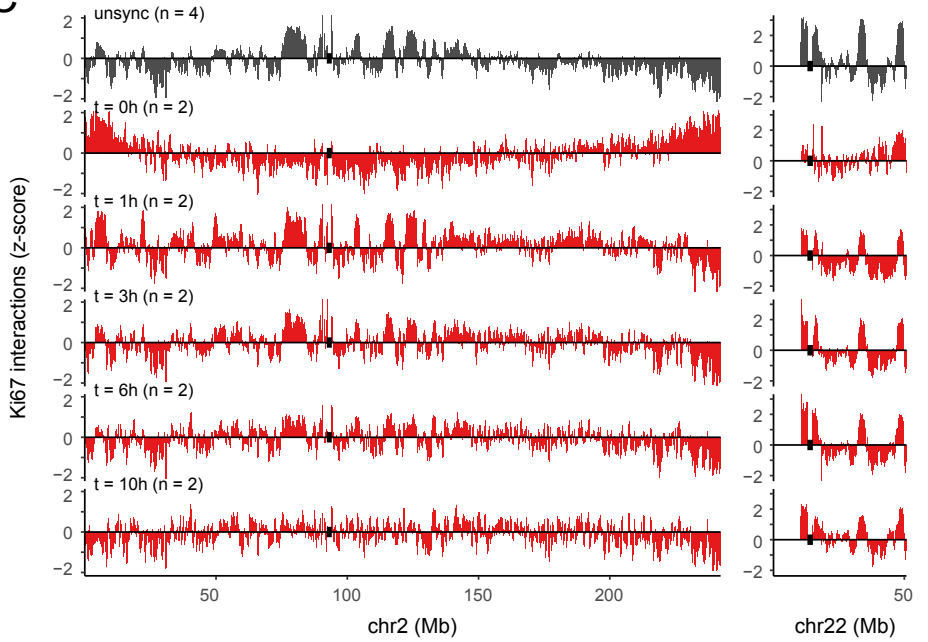

chr22 (Mb)

D

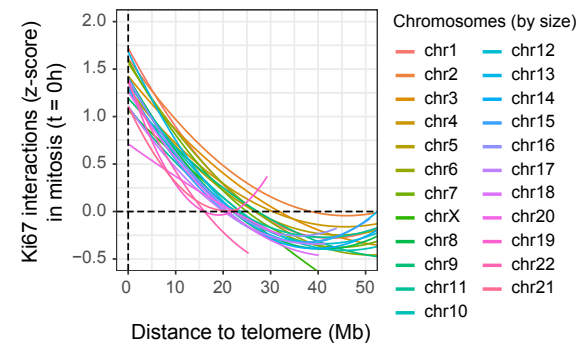

$E$

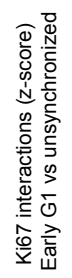

$\mathrm{F}$

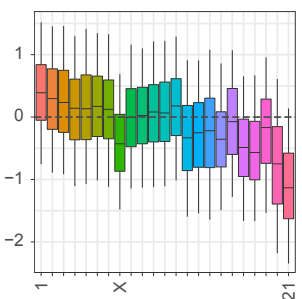

Chromosome (by size)

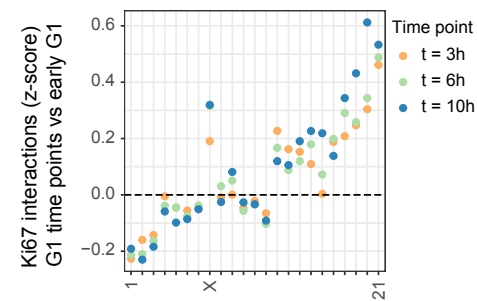

Chromosomes (by size)

\section{Fig 2. Cell cycle dynamics of Ki67 interactions}

(A) Representative confocal microscopy sections of hTERT-RPE cells processed with Ki67 pA-DamID, following synchronization with a mitotic shake-off ( $\mathrm{t}=0 \mathrm{~h}$, see Methods) and time points after replating of the synchronized cells. Cells were stained for Ki67 and labeled with m6A-Tracer protein. Scale bar: $5 \mu \mathrm{m}$.

(B) Quantification of m6A-Tracer and Ki67 antibody enrichment near Ki67 domains (interpreted as PNBs / nucleoli) in synchronized hTERT-RPE cells, as described in (Fig 1C). Instead of individual cell traces, the 95\%-confidence interval of the mean is added as shaded area. Results are combined from three (unsynchronized cells) or one (synchronized cells) biological replicates.

(C) Ki67 interactions profiles for the synchronized hTERT-RPE cells. Two representative chromosomes were visualized to highlight the differences between large (chr2) and small chromosomes (chr22). Log2-ratios were converted to $z$-scores to correct for differences in dynamic range between conditions and replicates. Data profiles are averages of $n$ experiments and smoothed with a running mean across nine $50 \mathrm{~kb}$ bins. Centromeres are highlighted by black bars.

(D) Smooth loess curves of Ki67 interactions plotted against the distance to chromosome ends for cells synchronized in metaphase ( $\mathrm{t}=0 \mathrm{~h})$. Lines represent individual chromosomes.

(E) Chromosomal distributions of genome-wide differences between Ki67 interactions in early G1 $(\mathrm{t}=1 \mathrm{~h})$ and unsynchronized cells. Boxplots: horizontal lines represent 25 th, 50 th, and 75 th percentiles; whiskers extend to 5 th and 95th percentiles.

(F) Mean chromosomal differences in Ki67 interaction scores between interphase time points and early G1 cells $(\mathrm{t}=1 \mathrm{~h})$ are plotted for chromosomes sorted by size. 

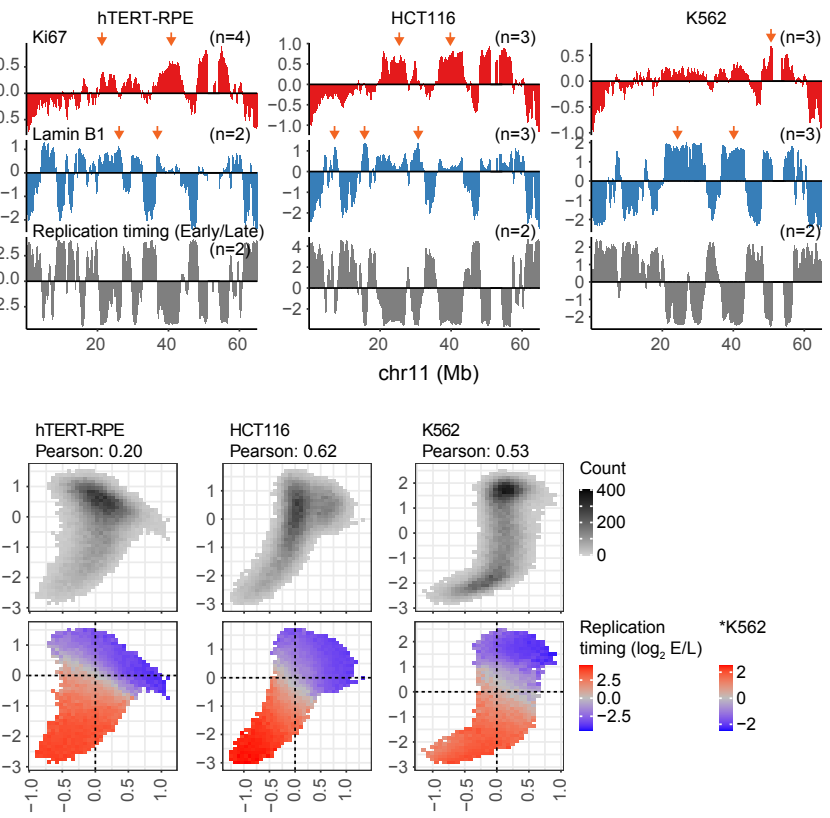

K562

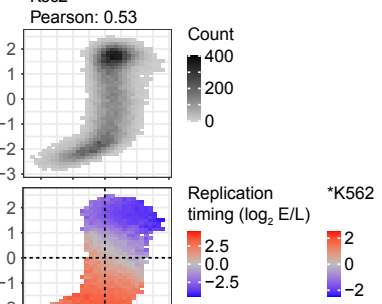

Ki67 interactions $\left(\log _{2}\right)$

\section{Fig 4. Ki67 and Lamin B1 together mark the late-replicating genome}

(A) Comparison of Ki67 interactions with Lamin B1 interactions (both log2-ratios) and DNA replication timing (Repli-seq log2-ratio of Early/Late) for a representative genomic region. Orange arrows highlight domains with high Ki67 and generally low Lamin B1 interactions, and vice-versa. Data profiles are averages of $\mathrm{n}$ experiments and smoothed with a running mean across nine $50 \mathrm{~kb}$ bins. Centromeres are highlighted by black bars.

(B) Binned scatterplots between Ki67 and Lamin B1 interactions (log2-ratios), showing the amount of genomic 50kb bins overlapping these points (top panels) and the mean replication timing score of overlapping genomic bins (log2 Early/Late) (bottom panels). Points are only shown with at least 10 overlapping genomic bins for a robust estimation of mean scores. A separate color bar is used for replication timing in K562 cells, which have a reduced dynamic range. Replication timing data were obtained from the 4D Nucleome data repository [36]. 
S-phase

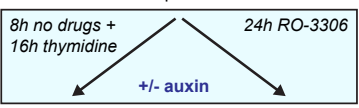

S-phase

(with mitosis)

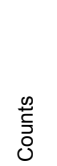

500

DAPI intensity (A.U.)

G2-phase (without mitosis)

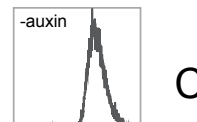

C

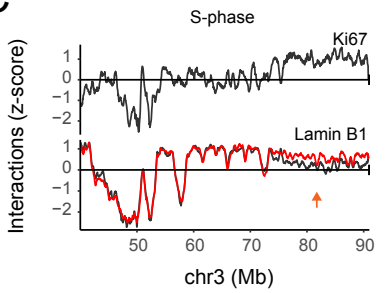

$\mathrm{F}$

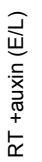

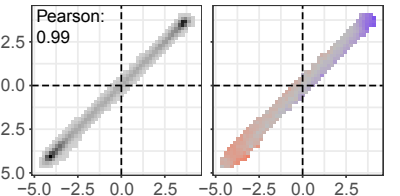

$\circ$ 응 $\vec{\circ}$ $R T$-auxin (E/L)

Count

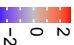

Ki67 interactions (z-score)

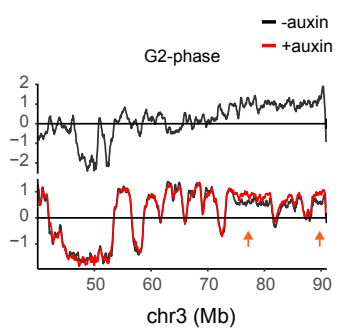

G

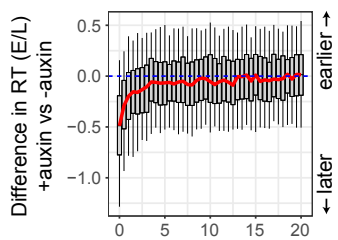

Distance to centromere $(\mathrm{Mb})$

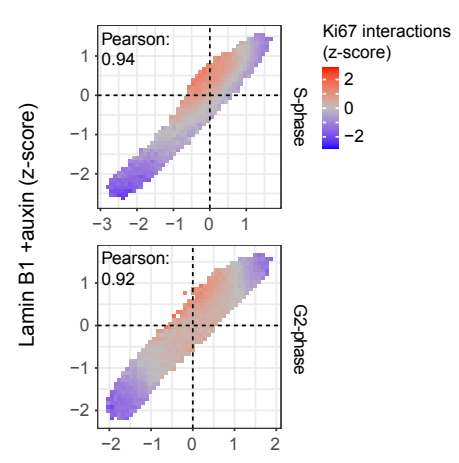

Lamin B1 -auxin (z-score)
$\mathrm{H}$

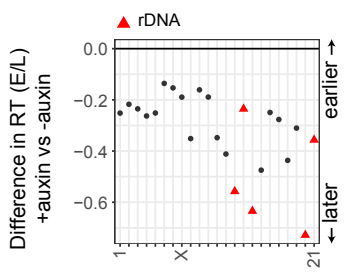

Chromosome (by size)

- -auxin

-2
$x i n$
$x i n$

chr12 (Mb)

\section{Fig 5. Effects of Ki67 depletion on Lamin B1 interactions and replication timing}

(A) Overview of the Ki67 depletion experiment in HCT116 Ki67-AID cells (top). Cells were either cultured with a double thymidine arrest to enrich for S-phase cells (which includes one cell division) or arrested in G2 by CDK1 inhibition immediately following an initial S-phase arrest (which prevents a cell division). FACS analysis confirmed successful synchronization and illustrates that Ki67 depletion does not affect this (bottom panels).

(B) Differences in mean Ki67 chromosome scores between S- and G2-synchronized HCT116 Ki67-AID cells.

(C) Representative genomic region showing the effect of Ki67 depletion on Lamin B1 interactions, for HCT116 Ki67-AID cells synchronized in S-phase (left panel) and G2-phase (right panel). Black and red lines represent conditions with normal and depleted Ki67 levels, respectively. Ki67 interactions were only profiled at non-depleted levels. Orange arrows highlight regions with high Ki67 interactions that gain Lamin B1 interactions upon Ki67 depletion. Data profiles are averages of 2 experiments and smoothed with a running mean across nine $50 \mathrm{~kb}$ bins. The centromere is located just to the right of the depicted region.

(D) Binned scatterplots of Lamin B1 interactions at in HCT116 cells with normal versus depleted Ki67 levels, as described in (Fig 4B). Bins are colored by mean Ki67 interactions in S-phase (top panels) and G2-phase (bottom panels). Signals are smoothed with a running mean of three $50 \mathrm{~kb}$ bins to reduce noise in the data.

(E) Representative genomic region showing the effect of auxin-mediated Ki67 depletion on (S-phase) Lamin B1 interactions and replication timing (log2 Early/Late) in HCT116 Ki67-AID cells, as described in (C). Orange arrows highlight domains with gained Lamin B1 interactions and delayed replication timing; purple arrows highlight domains with gained Lamin B1 interactions but unaffected replication timing.

(F) Binned scatterplots (as in (D)) of replication timing (RT, E/L) in cells with normal versus depleted Ki67 levels, colored by the density of overlapping $50 \mathrm{~kb}$ bins (left panel) and mean Ki67 interactions at normal Ki67 levels (right panel).

(G) Distribution of differences in replication timing (RT) nearby centromeres. Boxplots: horizontal lines represent 25th, 50th, and 75th percentiles; whiskers extend to 5th and 95th percentiles.

(H) Mean difference in replication timing (RT) within $2 \mathrm{Mb}$ from the centromere of each chromosome. rDNA-containing chromosomes are highlighted in red. 

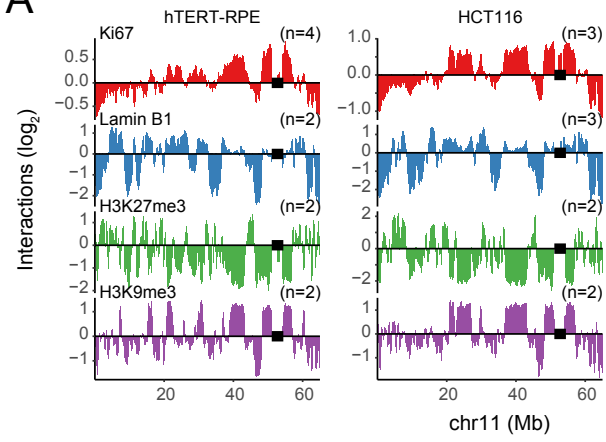

B

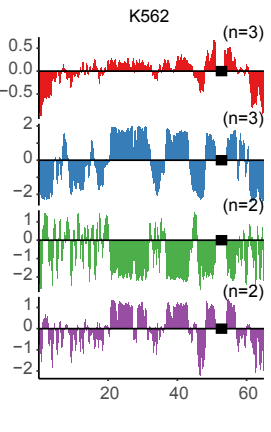

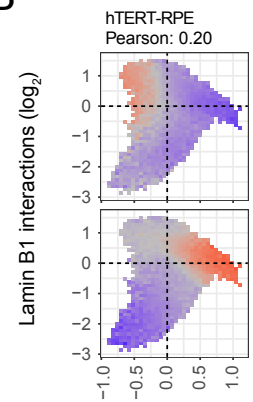
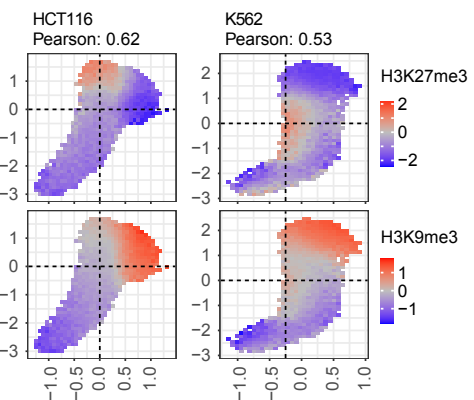

Ki67 interactions $\left(\log _{2}\right)$
C
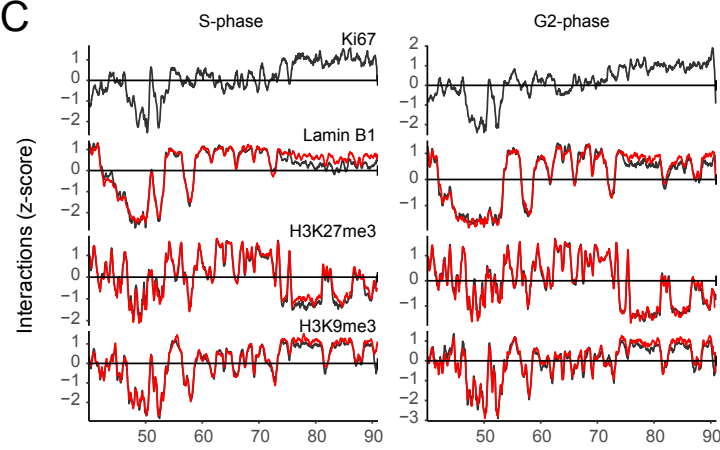

- -auxin

$\operatorname{chr} 3(\mathrm{Mb})$
$\mathrm{D}$
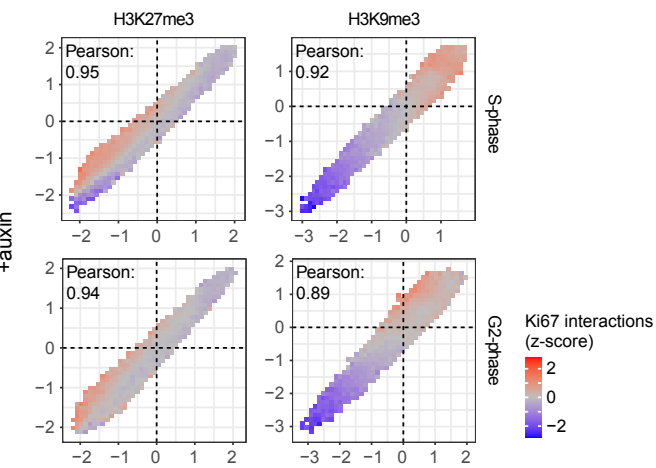

-auxin

\section{Fig 6. Comparison of the Ki67 and Lamin B1 balance with H3K27me3 and H3K9me3}

(A) Comparison of Ki67 interactions with Lamin B1 interactions and H3K27me3 and H3K9me3 histone modifications for a representative genomic region. Data profiles are averages of $\mathrm{n}$ pA-DamID experiments and smoothed with a running mean across $950 \mathrm{~kb}$ bins. Centromeres are highlighted by black bars.

(B) Binned scatterplots of Ki67 and Lamin B1 interactions as in (Fig 4B), colored according to the mean H3K27me3 and H3K9me3 signals.

(C) A similar figure as (Fig 5B), but additionally showing the effect of Ki67 depletion on H3K27me3 and H3K9me3.

(D) Similar plots as (Fig 5D), but instead showing H3K27me3 and H3K9me3 at normal and depleted Ki67 levels. Bins are colored by the Ki67 interactions in cells with normal Ki67 levels. 


\section{A}

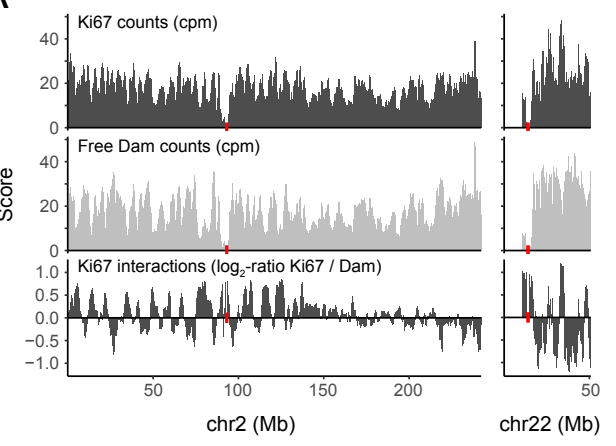

B
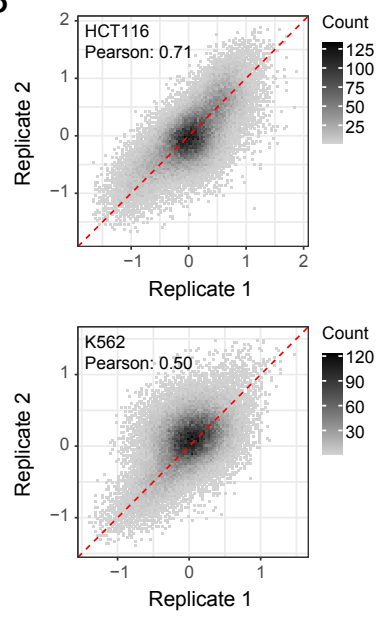

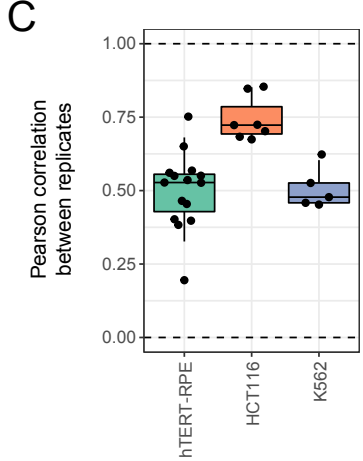

$\mathrm{E}$
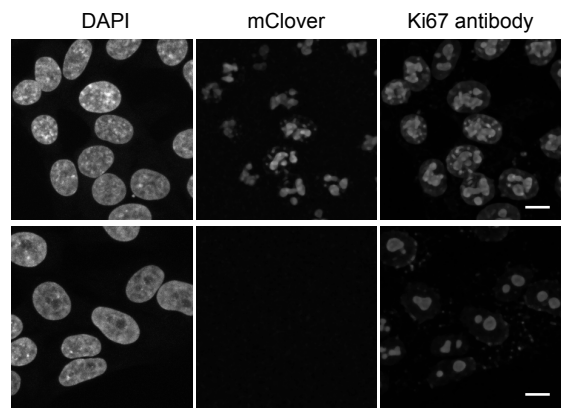

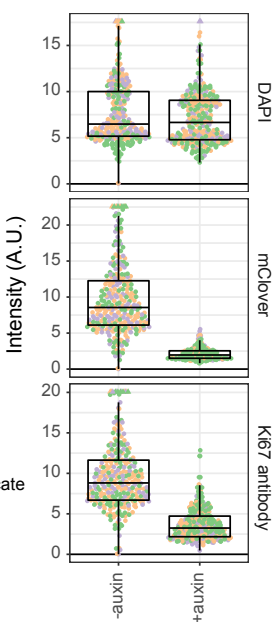

$\mathrm{F}$
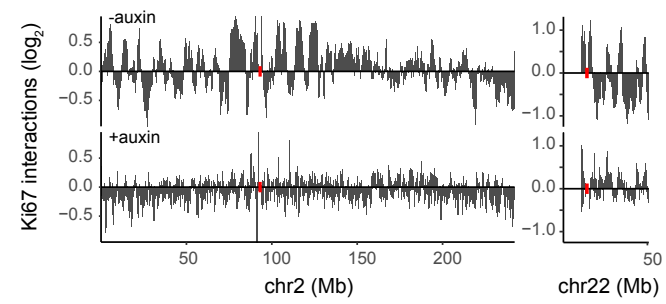

G

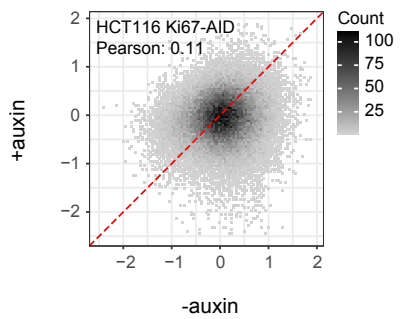

Fig S1. Additional information and controls for Ki67 pA-DamID

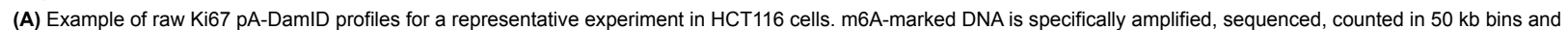

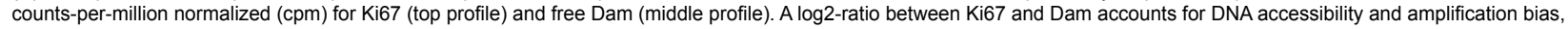

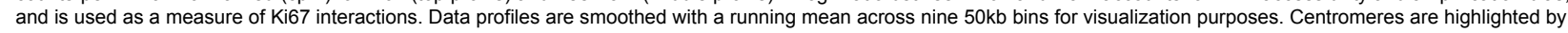
red bars.

(B) Replicate experiment correlations for Ki67 interactions (log2-ratios) measured in unsynchronized HCT116 cells (top panel) and K562 cells (bottom panel).

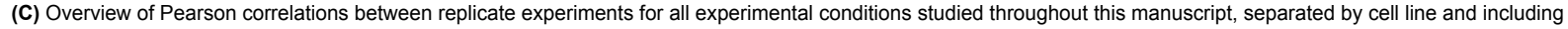
perturbation experiments.

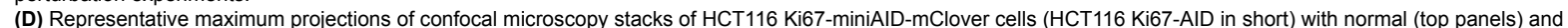

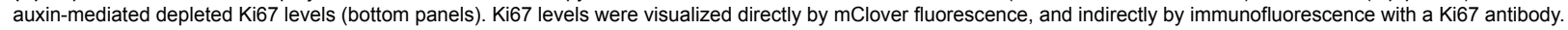
Scale bar: $10 \mu \mathrm{m}$.

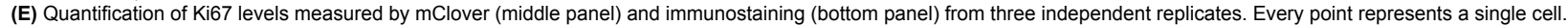

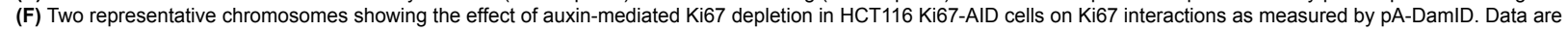
averages of two biological replicates and smoothed with a running mean across nine 50kb bins. Centromeres are marked by red bars.

(G) Correlation of all $50 \mathrm{~kb}$ genomic bins from profiles described in (F). 


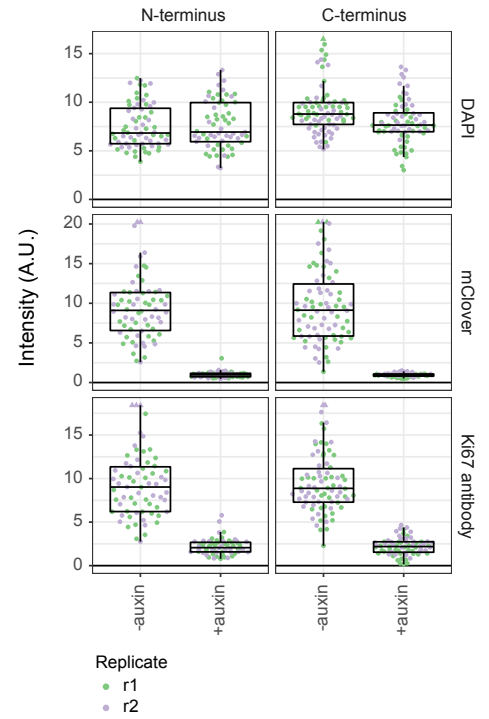

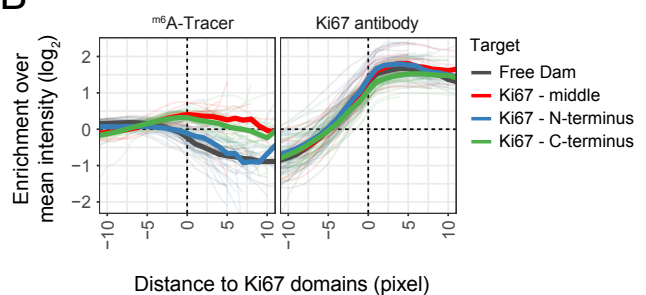

C

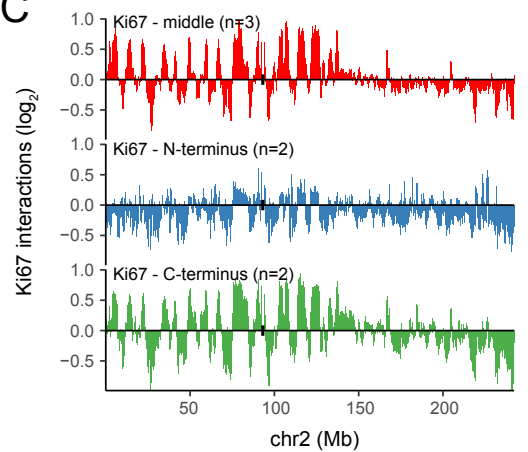

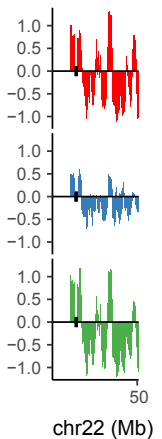

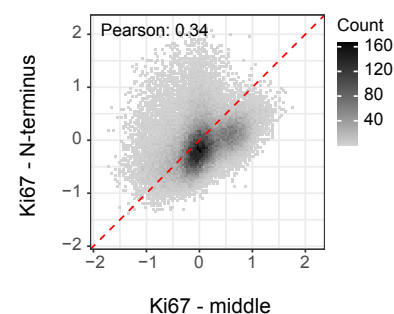

$\mathrm{E}$

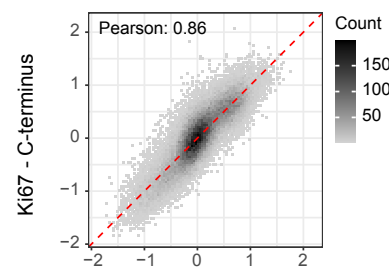

Ki67 - middle

\section{Fig S2. Ki67 interactions are reproducible between antibodies}

(A) Quantification of Ki67 levels as described in (Fig S1D-E) based on mClover and Ki67 immunostaining signals in HCT116 Ki67-AID cells using different Ki67 antibodies. For this experiment, single confocal microscopy sections from the middle of cell nuclei were used rather than maximum projections from entire nuclei. Results are combined from two biological replicates.

(B) Quantification of m6A-Tracer enrichment around Ki67-marked nucleoli, as described in (Fig 1B-C). Results are combined from one (middle-targeting antibody) or two (N- and C-terminus antibodies) biological replicates. Data from Fig 1C are not included here.

(C) Ki67 interactions on two representative chromosomes as determined by pA-DamID using three different Ki67 antibodies. Data profiles are averages of $\mathrm{n}$ biological replicates and smoothed with a running mean across nine $50 \mathrm{~kb}$ bins. Centromeres are highlighted by black bars.

(D-E) Correlation of all $50 \mathrm{~kb}$ genomic bins between Ki67 interactions profiled with the middle-targeting antibody and the N- (D) and C-terminus antibodies (E). 


\section{A}
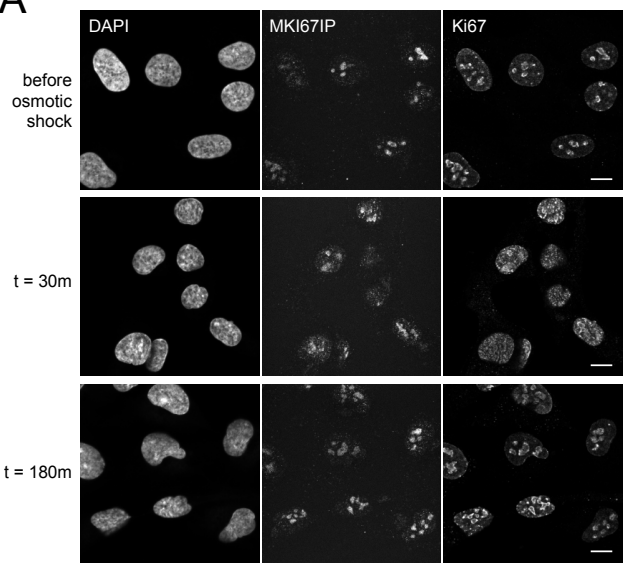

C

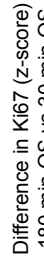

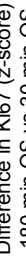

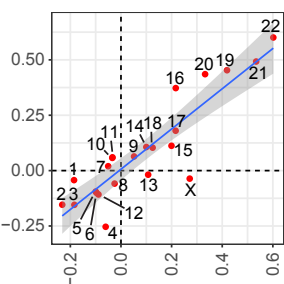

Difference in Ki67 (z-score) Late G1 vs Early G1
B
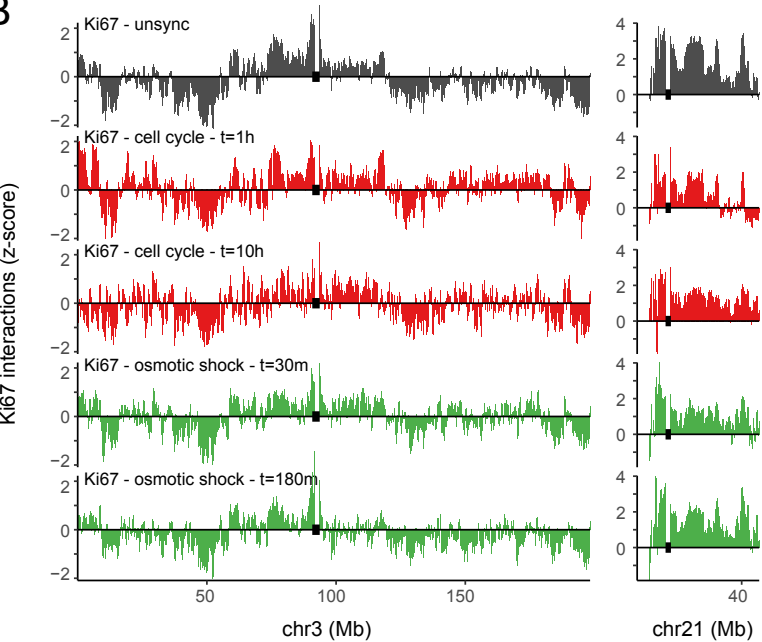

chr21 $(\mathrm{Mb})$

$E$
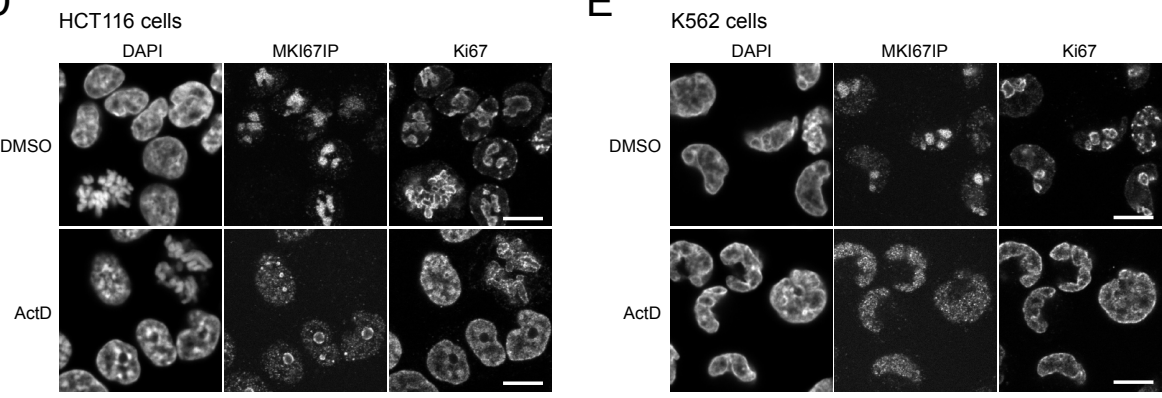

\section{Fig S4. A short osmotic shock recapitulates Ki67 maturation from PNBs to nucleoli}

(A) Representative confocal microscopy sections of hTERT-RPE cells before a 15-minute osmotic shock (0.2x PBS) (top panels), and after 30 and 180 minutes of recovery in complete medium (middle and bottom panels). Formaldehyde-fixed cells were stained for MKI67IP and Ki67. Scale bar: $10 \mu \mathrm{m}$.

(B) Representative chromosomes showing Ki67 interactions in unsynchronized hTERT-RPE cells, two interphase time points (data are the same as in Fig 2C) and the two time points following osmotic shock. Note that the regions that change Ki67 interactions during interphase show the same trends after the osmotic shock. Data profiles are averages of two experiments and smoothed with a running mean across nine $50 \mathrm{~kb}$ bins. Centromeres are highlighted by black bars.

(C) Changes in chromosomal distributions of Ki67 are similar for progression in $\mathrm{G} 1$ phase (x-axis: difference in mean interactions per chromosome between $10 \mathrm{~h}$ and $1 \mathrm{~h}$ after mitosis) and upon recovery from osmotic shock (y-axis: difference in mean interactions per chromosome between 180 min and 30 min after termination of the osmotic shock (OS)). (D-E) Representative confocal microscopy sections showing the effect of ActD on nucleolar morphology in HCT116 (D) and K562 cells (E), as described in (Fig 3A). Scale bar: $10 \mu$ $\mathrm{m}$. 
$A$

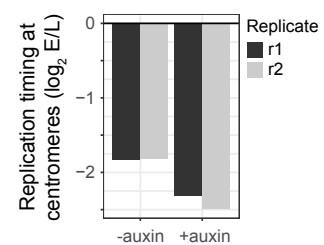

Replicate experiment
B

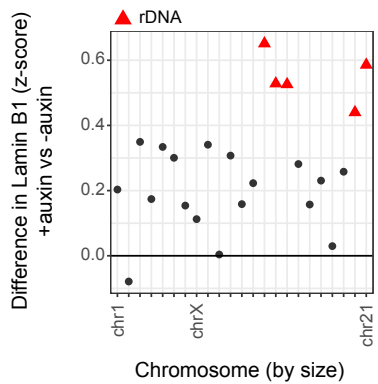

C

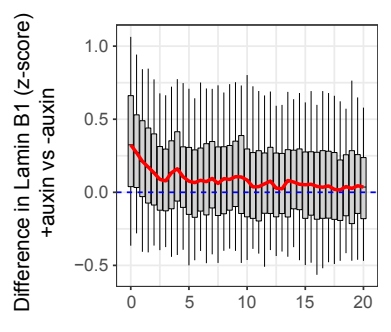

Distance to centromere $(\mathrm{Mb})$
D

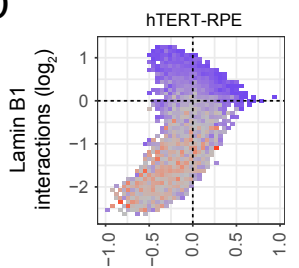

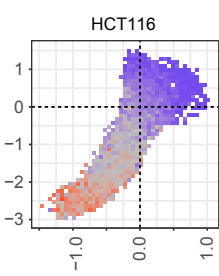

Ki67 interactions $\left(\log _{2}\right)$

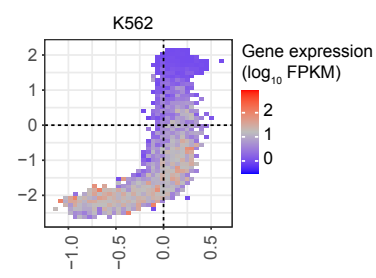

$E$

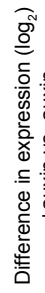

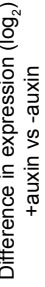

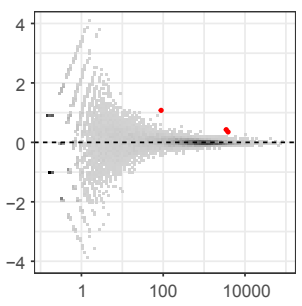

Mean expression (cpm)

- Significant $(n=3)$
F

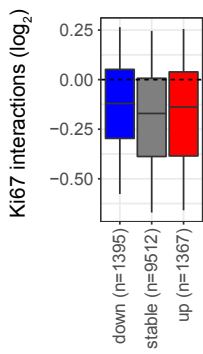

hTERT-RPE expression class

\section{Fig S5. Loss of Ki67 interactions does not correlate with gene expression changes}

(A) Bar plot showing average replication timing (log2-ratio Early/Late) for centromeres. All reads were selected that aligned to regions marked as centromeres (UCSC Table browser; hg38 centromeres) regardless of their mapping quality and, following counts-per-million normalization, the E/L ratio was calculated between the total centromeric counts. Between $3-5 \%$ of the late-fraction mapped to centromeres.

(B-C) Similar plots as (Fig 5G-H), but showing differences in Lamin B1 interactions instead.

(D) Similar plot as (Fig 4B), but instead highlighting for every gene the expression level (in log10 FPKM). Expression data are used from [30, 65-70] (see Methods).

(E) Scatterplot of mean gene expression (counts per million; cpm) versus expression difference (log2-fold change) following auxin-mediated Ki67 depletion in HCT116 Ki67-AID cells. Significant genes (Benjamini-Hochberg adjusted p-value <0.05) are highlighted in red.

(F) Boxplots showing Ki67 interactions for differentially expressed genes following Ki67 depletion in hTERT-RPE cells. Gene expression calls are from [30]. Boxplots: horizontal lines represent 25 th, 50 th, and 75 th percentiles; whiskers extend to 5 th and 95 th percentiles. 